International Research Journal of Materials Sciences and Applications

(ISSN:2575-1034)

\title{
Vinyl Ester/Glass Microballoon Syntactic Foams with Low Density
}

\author{
Zulzamri Salleh $^{1,2}$, Md Mainul Islam ${ }^{* 1}$, Jayantha Ananda Epaarachchi ${ }^{1}$, Haibin $\mathrm{Su}^{3}$
}

${ }^{1}$ Centre for Future Materials, Faculty of Health, Engineering and Sciences, University of Southern Queensland, Toowoomba, Queensland 4350, Australia; ${ }^{2}$ Universiti Kuala Lumpur, Malaysian Institute of Marine Engineering Technology, 32200, Lumut, Perak, Malaysia; ${ }^{3}$ Division of Materials Science, Nanyang Technological University, 50 Nanyang Avenue, Singapore 639798.

\section{ABSTRACT}

In this study, the synthesis and characterization of vinyl ester/ glass microballoon syntactic foams with different weight percentages are investigated. Both the tensile and compressive properties of the syntactic foams are characterized. Generally, the results show that the compressive strength and moduli of several syntactic foam compositions are comparable to those of pure vinyl ester as a matrix resin. Due to the lower density of syntactic foams, the specific compressive properties of SCFT-01 (2wt.\%) are higher than all other specimens and closer to neat resin. Similar trends are also observed in the tensile properties. The results show that syntactic foams with vinyl ester matrix possess strength behaviour that varies with different content of glass microballoons. This difference is related to the possibility of particle fracture in the stress range where modulus is calculated from the compressive stress-strain curves. In addition, tensile modulus is found to be $70-80 \%$ higher than the compressive modulus for all syntactic foam compositions, but both of them are still lower than neat resin. The results also show that the content of glass microballoons in the syntactic foams should be controlled to obtain a good combination of compressive strength and tensile strength. The reasons are discussed in detail.

Keywords:

Syntactic foam, composite, glass microballoon, resin, wall thickness

\section{${ }^{*}$ Correspondence to Author:}

Dr. Md Mainul Islam, Senior Lecturer, Centre for Future Materials and School of Mechanical \& Electrical Engineering, Faculty of Health, Engineering and Sciences, University of Southern Queensland, Toowoomba, Queensland 4350, Australia. Tel: +61746311388 Fax: +61746312526 E-mail: Mainul.Islam @ usq.edu.au

How to cite this article:

Salleh et al., Vinyl Ester/Glass Microballoon Syntactic Foams with Low Density. International Research Journal of Materials Sciences and Applications, 2017; 1:1.

\section{eSciencePublisher}

eSciPub LLC, Houston, TX USA. Website: http://escipub.com/ 


\section{Introduction}

Nowadays materials possessing a lower density and higher performance in mechanical properties is a priority for engineering structures, especially marine engineering structures. These features can only be found in close-cell foam such as syntactic foam glass microballoons. The foam is made from a special materials combination matrix (binder) and microspheres hollow sphere (filler) with a formal structure such as that found in cellular and solidified liquid. Syntactic foam has provided many benefits in the development of aircraft, spacecraft and application naval structures [1-3]. It can also be used in the application of electronic materials and packaging [3]. Syntactic foam also has good thermal insulation [4]. Depending on the application to be made, the characteristics of the matrix material, microballoons, fragments of microballoons, and microballoons density are important aspects in making syntactic foam can be varied. In the construction industry, syntactic foams have been used as imitations of wood and marble [3] which are ideal core materials due their effective shear stiffness and strength. Vasanth et al. [5] found that, products which requested for lightweight materials it has important to consider for the wall thickness and volume fraction of microballoons. From the study by Gupta et al. [6], compressive properties are not found to be significantly improved in gradient structures fabricated with a variation in microballoon volume fraction. Gupta et al. [6] fabricated syntactic foams using glass microballoons and studied the compressive properties with a variation in microballoon volume fraction.

Generally, glass microballoons use a polymer resin such as epoxy resin, cyanate ester, polypropylene, polysialate and vinyl ester and others polymer resins [7] as matrix materials. Of the polymers matrix materials mentioned above, epoxy matrix syntactic foams have been widely studied due to the extensive use of epoxy resins in aerospace applications [8-13]. When demand for this resin is comparatively high, the price rises in market of composites and alternative resins which has similar behaviour but without compromised mechanical properties must be found. Vinyl ester is a better choice in term of competitive price and is widely used in marine applications [14-16]. Therefore, investigating the properties of vinyl ester matrix syntactic foams and devel- oping structure-property correlations for these materials are important challenges.

In previous works, many researchers were interested in the properties of foams particularly on the compressive strength and tensile strength. When compressive behaviour is observed, all syntactic foam compositions show a stress plateau [17]. Similar results have been observed for epoxy and aluminium matrix syntactic foams [18, 19]. The investigation on the effects of the microballoon size on the compressive strength is beneficial for future engineering [20]. From the research, it was found that smaller microspheres ensure higher compressive strength than large ones because they contain fewer flaws in their microstructure. Palmer et al. [21] proved that larger microspheres contain more porosity in their walls and more flaws in their microstructure than the smaller ones.

The properties of glass microballoons will also be reported in this paper, as they contribute to the remaining porosity in the syntactic foam. The compressive strength of different weight percentage of composition were investigated including their plateau and tensile strength characteristics are also discussed. Based on the previous report also found that, the compression behaviour of foams are related with either microsphere/matrix adhesion or on the wall thickness of the hollow microspheres along with fracture analysis [22, 23]. This study also reported and discussed the porosity content in the glass microballoon/vinyl ester syntactic foams as well as void contents in the specimens. No reports were identified for this type of glass microballoons that have been used in the production of syntactic foams. Thus, the specific compressive including plateau stress and energy absorption for this type of glass microballoon is also reported and discussed in this paper.

Many researchers have proposed the use of glass microballoon because of their high specific strength and energy absorption properties [20]. A variety of syntactic applications have been explored in within their mechanical properties, such as tensile properties, the main focus for researcher. Hence, it is helpful to understand the role of microballoons and resin matrix in the failure behaviour of syntactic foams. Previously, compressive results have revealed that the strength and modulus of syntactic foams increase with an 
increase in density of microballoons [21], while tensile strength testing has shown a significant increase in the strength of syntactic foams with an increase in the volume fraction of glass microballoons [22, 23]. Although these mechanical properties have been studied extensively, the tensile properties of syntactic foam have not yet been established. Only a few reports have deal with the tensile and compression properties of syntactic foams [24-27] and it has now become necessary to characterise their tensile behaviour and failure characteristics.

This study focuses on the physical properties, compression properties and tensile properties as well as the fractured mechanism of glass microballoon/vinyl ester syntactic foams. Fifteen types of syntactic foams are fabricated using one type of microballoon in five different weight percentages (wt.\%) for both of tensile and compression testing. The tensile and compressive properties of these materials are characterized; including modulus, strength and fracture features. The effect of weight percentage and wall thickness of the microballoons on the mechanical properties of the composite is experimentally investigated. All the specimens are prepared using the open mould method and are tested using universal testing machine (UTM). In order to characterise the fracture mechanism of syntactic foams, microstructure analysis has been introduced with the use of a scanning electron microscope (SEM).

\section{Experimental Methods}

\subsection{Constituent Materials and Equipment}

The vinyl ester resin with the scientific name diglicidyl ether of bisphenol A-based resin and methyl ethyl ketone peroxide (MEKP) catalyst, used as the matrix material, were procured from the Australian company with wholly known as NOROX. The glass microballoons used in this study were non-porous in nature and are manufactured and supplied by Potters Industries Inc. It was trading under the name of Q-CEL Spherical (R) Hollow Microspheres with the part number 5020 .From the manufacturing data sheet, the physical shape of glass microballoon has spherical glass powder typed with chemically-stable fused-borosilicate glass and non-porous micro- sphere [28]. Fig. 1(a) shows the scanning electron micrograph of glass microballoons particles before incorporation with matrix resin used in this study. The mean inner diameter was calculated by taking the difference in the average true particle density of solid and hollow particles made up of the same material. The mean particle size distribution and bulk density of this particle supplied by the manufacturer have given as $72 \mu \mathrm{m}$ $(5-150 \mu \mathrm{m})$ and $110 \mathrm{~kg} / \mathrm{m}^{3}$, respectively.

Density of syntactic foam was measured using gas replacement according to ASTM D2840. The helium gas was supplied in a cylinder tank with a multipycnometer unit (Quantachrome Instrument model). The measurement was set up at the room temperature condition of $25^{\circ} \mathrm{C}$. First of all, the specimens were grinded using a mortar and pestle to create small granular pieces. The pieces were placed into a multipycnometer cup before being placed in the measurement area. Fig. 1(b) shows the overview of multipycnometer unit used for density measurement

Compression testing (ASTM D695) was also performed using MTS test systems with the crosshead speed of $2 \mathrm{~mm} / \mathrm{min}$. From the output result, the two parameters of load and crosshead displacement were selected for data analysis and development of stress-strain curves. Fig.1 (c) shows the photo of one of the specimens during compression testing with the MTS machine.

Tensile testing (ASTM D638) was carried out using a computer controlled MTS Insight universal testing machine shown in Fig 1(d). At least three specimens of each type of composition glass microballoon for vinyl/ester syntactic foams were tested. Specimens were subjected to tensile loading at a cross speed $1.25 \mathrm{~mm} / \mathrm{min}$. Strain data were measured through an extensometer with a $25 \mathrm{~mm}$ gauge length. Load-displacement data obtained from the tests were used to calculate the strength, modulus and Poisson ratio's properties glass microballoon/vinyl ester syntactic foams.

\subsection{Fabrication of Syntactic Foam}

The fabrication of tensile specimens by steel mould into what is called a 'dog bone' shape have been used in this study. The compression specimens, were made with a PVC mould with the dimensions diameter, $\varphi=22.0 \mathrm{~mm} \times$ length, 
$\mathrm{L}=44.0 \mathrm{~mm}$. First of all the moulds must be cleaned using acetone and the surface coated with mould wax to ensure that the syntactic foams can be removed easily. The commonly used mould releasing agent of a silicone gel type, was employed particularly for the stainless steel mould [27].

Stir mixing is a well-known method for fabricating syntactic foams. Mixing with a glass rod is common nowadays, but the stir mixer machine using a stir magnetic bar as a mixer is a better performer or using the ultrasonic machine for timely manner consuming. The mixture can also be manually shaken to gelatinise the starch and microballoons in a container [26]. The manually mixing or conventional method needs to be followed carefully to avoid the breakage of microballoons [4]. This synthesis method consists of mixing measured quantities of glass microballoons in the resin and mixing them until a slurry of uniform viscosity is obtained. The mixing time is approximately is between 4 to 5 minutes. Stirring time should be increased for a higher weight percentage of microballoons to ensure uniform distribution of glass microballoons in the resin. As the amount of the glass microballoons increases, the viscosity also increases and the mixtures develop putty-like consistency and difficult to homogeneity when using the mixer machine.

A putty-like consistency is impossible to stir because it became the dough and stick to each other. Therefore, the selection of composition glass microballoons in weight percentage is also important to ensure that this phenomenon can be avoided during sample preparation. Then, all the mixtures are transferred and cast into the moulds for tensile and compressive testing used after it wax with silicone gel (mould releasing agent). The sample along with the mould was allowed to cure for 24 hours at room temperature and then the samples were removed from the moulds. At the final stage of fabrication, all the specimens were post-cured at $60-80^{\circ} \mathrm{C}$ for 4 hours in a hot air oven.

\subsection{Syntactic Foam Density}

Constant temperature was used to determine the densities of syntactic foam by using Boyle's Law where the pressure and volume of the gas are important parameters in ideal gas law [29]. According to this law, the pressure of the gas is proportional to the volume of gas, then the multiplication of pressure $\left(\mathrm{P}_{\text {gas }}\right)$ with volume of the gas $\left(\mathrm{V}_{\text {gas }}\right)$. The above parameters can be connected by using $R$ as Boltzmann constant and $T$ is remains constant and $n=1$ (He gas). Finally, this will determine the initial values for the pressure and volume of syntactic foam while final values are considered as the pressure and volume of the gas, respectively. Therefore, it is important to determine the volume of the syntactic foam in order to calculate the density of syntactic foam when mass is divided by their volume itself. Then to measure the volume fraction for each of the specimens, the rule of mixtures was implemented using Equation (1) below,

$$
\text { Volume fraction, } \mathrm{Vf}=\frac{\mathrm{Wf}}{\mathrm{Wf}+(1-\mathrm{Wf}) \frac{\rho f}{\rho m}}
$$

Where Wf is weight of filler/glass microballoon, $\rho f$ is density of filler/glass microballoon and $\rho m$ is density of matrix/vinyl ester resin. Equation (1) is very useful for measuring the density of composites particularly for syntactic foam [26]. The compositions of glass microballoons in weight percentage (wt.\%) in this study are $2 \mathrm{wt} . \%, 4$ wt. $\%$, $6 w t . \%, 8 w t . \%$ and $10 w t . \%$. The density of syntactic foam was measured by using a crushed microballoons sample in a Quanta-chrome Ultra pycnometer. The density of syntactic foam also affected the mechanical properties particularly porosity contents in the syntactic foam also need to be investigated in this study.

\subsection{Syntactic Foam Porosity}

The calculation of the parameters related to wall thickness, $\omega$ such as radius ratio, $\eta$ and volume of porosities as shown below. The ratio of inner, $r_{i}$ to outer, $r_{0}$ radius of microballoons, called radius ratio $\eta$, is also reported in Table 1 . The radius ratio is approximately calculated based on the standard glass density of $2540 \mathrm{~kg} / \mathrm{m}^{3}$ or on the mean particle density [24]. The diagram for outer and inner radii together with wall thickness can be seen in Fig. 2(a). It is clear that if $r_{0}$ is the same, any difference in the $\rho_{g m}$ is caused by a difference in their $\eta$. Two types of porosity occur in syntactic foams; namely microballoons porosity and matrix porosity [25]. The empty microballoon volume enclosed within the microballoons 
gives rise to microballoon porosity which is the closed cell porosity required to reduce the density of the syntactic foam material called volume cavity. The contents of matrix porosity of the desired closed cell microballoon in syntactic foams can be defined at Equation (2),

$$
V_{p, g m}=V_{g m} \times V_{c, g m}
$$

Where, $V_{\mathrm{gm}}$ " " = volume of glass microballoons in syntactic foam, volume cavity

$$
V_{c, g m}=\eta^{3} \text {, radius ratio } \eta=\left(1-\frac{\rho_{s f}}{\rho_{g m}}\right)^{\frac{1}{3}}, \eta=1-\frac{\omega}{\mathrm{r}} \text {, }
$$

$\rho_{\mathrm{gm}}$ density of glass microballoon, $\rho_{\mathrm{sf}}$ density of syntactic foam, wall thickness of glass microballoon [30] and $r$ is the average size of the glass microballoons.

The second type of porosity is matrix porosity which occurs because of the entrapment of air in the syntactic foam structure during the foam synthesis as shown in Fig. 2(b). This structure distribution especially for matrix porosity, depends on the contents of matrix porosity itself. During the mixing of the resin with microballoon, entrapment of air is inevitable leading to voids in syntactic foams.

\subsection{Mechanical properties}

The tensile behaviour of the syntactic foam will be discussed further in Section 3.3. Fig. 3 shows a typical tensile stress-strain curve for the syntactic foam used in this work. The graph shows that it exhibited linear behaviour, hence for each stress-strain curve, tensile modulus, tensile stress and strain at maximum peak can be determined. The stress-strain curve for compressive testing also was revealed in Fig. 3 . It shows that the trend for mean plateau was observed after achieving the maximum stress for syntactic foam until densification mode was detected. Densification can be described by three important regions of failure. These regions are called region (I)-initial linear deformation, region (II)-plastic mean plateau and region (III)-densification region. The details of this failure modes are also discussed in Section 3.3.
2.6 Effect of porosity on mechanical proper-

ties

Mechanical properties, particularly the compressive behaviour of syntactic foam, can be affected by porosity remaining in the composite materials. During the compression test, a severe shear crack was initiated and affected the glass microballoons and matrix resin. Gupta et al. [17] observed that crack formation is increased for a higher volume fraction of glass microballoons due to the brittleness of the composite. As a result, many of the glass microballoons and matrix resin were damaged during the testing and they was replaced to each other for example resin might be placed inside of broken glass microballoon to develop one of the porosity continent. In order to determine the relationship between the mechanical properties and porosity, Phang and Ding proposed the simple Equation (3) at below [31],

$$
\mathrm{E}_{\text {compressive }}=\mathrm{C}_{1}(1-\varphi)^{\mathrm{m}}
$$

Where $\mathrm{E}_{\text {compressive }}$ is compressive modulus elasticity, $C_{1}$ is constant, $(1-\varphi)$ is relative density with $\varphi$ is porosity syntactic foam and $m$ is positive exponential value. This linear equation can be solved by using logarithm function where $m$ is react as the gradient of the graph.

\section{Results}

\subsection{Density of Syntactic Foams}

The densities of syntactic foams for all specimens are presented in Fig.4. From the graph, it was observed that all the specimens show a decrease in their densities when the glass microballoon contents is increased. The data from the graph was calculated by using the rule of mixtures for Equation (1). A summary of all specimens is can also described in Table 1. All the specimens were as Pure VE, SCFT-01 (2 wt.\%), SCFT-02 (4 wt.\%), SCFT-03 (6 wt.\%), SCFT-04 (8 wt.\%) and SCFT-05(10 wt.\%). Generally, the density of syntactic foam becomes lighter when the glass microballoon content in the matrix resin is increased. It occurred due to the glass microballoon filling the gap between the foam and matrix resin. As the result, it is shown that the rule of mixtures is supported and followed in this 
study. From the graph, the specimen SCFT-01 (2 wt.\%) with the volume fraction $\left(V_{f}\right) 4 \%$ has a higher density but it is still lower than pure VE density. While the specimen with low density such as 10 wt $\%$ with the volume fraction $8 \%$ has low densities in their composition and might be created voids or porosity in the syntactic foam which will be discussed in Section 3.2. In addition, the glass microballoons also contributed to the weight of syntactic foam where the filler percentage is higher than resin. Another contribution to this behaviour also comes from glass microballoon debris in the matrix resin which will also be discussed further in Section 3.2.

From the above graph, the linearity of gradient shows decreased and the pattern is consider as particularly for composite materials which their densities were slightly decreased in order to achieve the low density behaviour. This characteristic was also been revealed by Swetha and Kumar [30] in their research where the density of syntactic foam decreased when the glass microballoon in the epoxy matrix resin increased for three different types of filler. The syntactic foams showed a linear decrease in density with an increase in the weight percentage of glass microballoons as filler incorporated into the vinyl ester resin. This behaviour is also reported elsewhere as the foamy nature of material [32]. This result also supports previous reports that showed a trend in decreased density [18, 33]. Therefore, the trend shows that the density of syntactic foam is proportional with the volume fraction of glass microballoon contents and is considered as evidence proving that the rule of mixtures for the composite materials in this study.

\subsection{Composite Porosity and Voids}

The fabrication of syntactic foam in this study used the open casting method. Hence, the surface of specimens has open bubbles and is rough with an irregular pattern because of the mixing of glass microballoon/vinyl ester and the MEKP dispersed with gas due to chemical reaction. This casting method is suitable for the fabrication of syntactic foams especially to release gas into the air. A similar method has been used in previous work [17]. In order to eliminate the rough surface at the external area of the syntactic foam during the compression testing, they surface must be grinded with a grinder machine to ensure flatness surface. Surface flatness is necessary because otherwise the mechanism of the compressive will be compromised test process and, as a result, the data will be unsatisfactory.

For the internal area of syntactic foam, the porosity and voids were randomly distributed at all surfaces. The mechanical properties of syntactic foam can be affected by porosity. Porosity can be identified as one of two types - type I occurs internally in microballoons while type II is matrix porosity due to air entrapped in the resin during the fabrication process as explained in detail in the published papers [2, 29, 34]. The voids also considered as one of type II matrix porosity when it was mentioned in previous literature [17]. Fig. 5 (a) shows that the total porosity content is increased while glass microballoons contents is increased in syntactic foams. The solution for Equation (10) gives the positive exponents for both linear graphs cavity and matrix porosities meet with cross to each other at gradient $m$ indicate that relative density porosities with 0.88 shows at Fig. 5 (b) for details. As a result, it is shown that, on average $12 \%$ contain both porosities for all specimens but voids are remain in all specimens because it cannot be avoided. Generally, if the specimens have increased glass microballoon content in syntactic foams, their porosity content increased which affects the mechanical properties.

The particle distribution including their porosities in the vinyl ester/glass microballoon syntactic foams in this study can also be seen by using a SEM machine shown in Fig. 6(a). Table 1 also shows the parameters of porosity and voids coexisting in the vinyl ester/glass microballoon syntactic foam including their wall thickness and radius ratio. Generally, the radius ratio and wall thickness are not much different when compared for all specimens. However, both the cavity porosity and matrix porosity have a significant effect on the development of syntactic foams. The specimen for SCFT-01 have less cavity porosity and matrix porosity with $57 \%$ and $16 \%$, respectively. Even though specimen SCFT-02 have a lower content of cavity porosity, matrix porosity is still highest when compared with SCFT-01. While the highest content for both porosities was led by specimen SCFT-05 which was more than $10 \%$ higher compared to SCFT-01. It might be 
caused by the huge quantity of fractured glass microballoons which became a debris as filler in the syntactic foam. Void contents were also present in all specimens which contributed to the lower density of the syntactic foam and slightly increased when glass microballoon content was increased.

The void contents were also calculated based on Equation (3) and presented in Table 1. Generally, the void contents are expected to increase when more glass microballoons are added to the syntactic foam from approximately $2.7 \%$ to $3.8 \%$ which is also supported by Fig. 6(b). This can happen during the fabrication of the syntactic foam using a conventional mixer $[4,26]$. The debris of the glass microballoons clearly shows this in Fig. 6(b).

A similar phenomenon can also be observed in this study, when the void and porosity contents are increased while glass microballoon content is increased during the manufacture of the syntactic foams [2, 29, 35]. Bibin et al. [29] explained that this discrepancy can be explained due to the partial distribution of resin and microballoons during compression moulding in the case of resin-rich systems. The manufacturing process can be improved by reducing the number of voids when releasing the bubbles or gas from the chemical reaction of MEKP. This can be made by using infusion methods.

The enlarged image of both porosities (type I and type II) can be seen clearly in Fig. 6(c). Table 1 also shows that the percentage of this type of porosity is increased from $33 \%$ to $53 \%$ when it is calculated here using the Equation (2). In some cases, the porosity should there be words embedded with small size of glass microballoon and filled with matrix resin also can be seen in Fig. 6(d). This type of matrix porosity occurred in specimens SCFT-02 to SCFT-05 and can be estimated using Equation (9). It was increased approximately on average with $1.5 \%$ to $2.0 \%$ individually. Hence, the total porosity also increases when the proportion of glass microballoon contents in the syntactic foam increases.

In this study, it can be seen that the volume of the both types of porosity have been calculated and the results show an increasing trend while the glass microballoon content increases. It is also revealed that the lower density is due to increas- ing the fracture of glass microballoon content, as shown in Fig. 6(a), especially for specimens SCFT-04 with 8 wt.\% microballoons as support from the section 3.1. The remaining debris also contributed to the lower density, as shown in Fig. 6(c), for SCFT-01(2 wt.\%). A broken microballoon filled with resin and smaller size microballoons is shown in Fig. 6(d) for SCFT-02 (4 wt.\%). As a result, it is shown that the matrix resin is filled inside the glass microballoons particularly for SCFT-01 which also contributed to the higher density. This evidence also supports the explanation in Section 3.1.

This kind of characteristic which also affected overall weight of syntactic foam. It is also become the reason the vinyl ester resin as exhibited dominantly in the syntactic foams as a main matrix. This phenomenon can happen when glass microballoons is fractured during the composite processing and, as a result, their cavities can be filled with higher density materials including glass debris and resin [17]. Also the void percentage has been calculated assuming that the microballoons are not broken during moulding. However, the breaking of some microballoons takes place during stirring process as it which cannot be avoided.

\subsection{Mechanical Properties}

The representative compressive stress-strain curves for all types of vinyl ester/glass microballoons are presented in Fig. 7 (a). From the stress-strain profiles, both neat resin and syntactic foams show the same trend for a linear elastic region followed by a strain softening region that is characterized by a slight drop in stress. When the compression continues further, the stress starts rising again. The increase in stress is faster and significantly higher in the case of neat resin, whereas for syntactic foams it depends on the type and volume fraction of microballoons [18]. The compressive modulus values are measured as the slope of the initial linear region of the stress-strain curves, and are presented in Fig. 7 (a).

The compressive strength of composites is defined as the first peak in the engineering stressstrain curves at stage (I). This initial linear deformation region (I) is where stress increases linearly to the first peak (from this gradient line 
yield strength or Young's Modulus can be defined), followed by a plastic plateau stage (II) where stress slightly increases as the strain increases, then there is a densification stage (III) where stress raises sharply with strain increasing slightly. Swetha and Kumar also observed that all syntactic foam compositions show this stress plateau, which is a typical feature of most types of syntactic foams [29].

The tensile properties of the vinyl ester/glass microballoon syntactic foam for different compositions of glass microballoon content has been carried out. The representative stress-strain curves for vinyl ester/glass microballoon syntactic foam specimens are presented in Fig. 7 (b). These curves show a linear stress-strain relationship, immediately followed by brittle fracture. The stress-strain curves for other types of syntactic foams also showed similar features $[17,25]$. The tensile characteristic values are listed in Table 1. The data including the CoV's (Coefficient of Variation's) for all specimens is presented in Table 1. The tensile strength from both data which is Table 1 and Fig. 7 (b) has shown similar tensile strength with smallest standard deviation. The tensile strength is led by pure vinyl ester at 39 $\mathrm{MPa}$ and all specimens show a decrease when the glass microballoon content increases.

The decreased tensile strength is observed starting from SCFT-01 at about 10\% from the neat resin then continues to reduce for SCFT-02 and no significant change occurs until SCFT-04 as shown in Fig. 7 (b). Similar characteristic also revealed for the reduction of strength value of the syntactic foam particularly tensile strength in matrix phase system which act as a load bearing phase as suggested by Wouterson et. al [9]. They tested glass microballoons in epoxy resin as matrix system. From their observation, it was found that the matrix-microballoon interface does not appear to be very strong in these composites, and the presence of a higher volume fraction of microballoons only reduces the volume fraction of epoxy resins in the structure, causing the lower strength of syntactic foams.

The tensile strength and modulus value of the vinyl ester matrix syntactic foam as a function of glass microballoon content are also shown in Fig. 8. Overall, the comparison of Young's modulus of all specimens shows that, specimen SCFT-2 is higher with content of $4 \mathrm{wt}, \%$ of glass micro- balloon at 9.92GPa. Generally, the modulus decreases while glass microballoon contents in the syntactic foam decrease in the syntactic foam. The reduction of modulus elasticity might occur due to the porosity contained in the syntactic foam and the majority of the broken glass microballoons are also filled with voids and porosity. In Table 1, it was also observed that modulus elasticity is reduced in the syntactic foam and it comparable with weight percentage or weight saving for low density syntactic foam. Gupta et. al [17] also had a similar reduction of elasticity in their research which is it can contribute to particularly for the substantial weight saving application. Huang and Gibson had also observed a similar trend for tensile modulus decreasing with an increase in glass microballoon content in syntactic foams [36]. In order to support the weight saving for low density syntactic foam, it is necessary to determine the specific strength of materials. From Fig. 8 (c) it is shown that specific tensile was led by SCFT-01 with $20 \%$ higher than other specimens, but with the trend showing a reduction among of them. While the specific modulus also was higher with SCFT-01 with a content of 2 wt.\% of glass microballoons and higher than pure VE. Therefore, in order to apply for marine application, weight sensitive concerns are very useful for this syntactic foam particularly SCFT01 it will achieve the weight saving matter.

The compressive maximum strength for syntactic foam is measured at the highest peak of the strain-stress curve shown at Fig. 8 (a). The results show that the compressive strength of syntactic foams decreases with an increasing content of glass microballoons particularly SCFT-01 which belong to the lower $\eta$. Gupta et al. [17] observed that if a radius ration glass microballoons of $\eta<0.955$ are used then the resulting syntactic foams would show substantial benefit in mechanical properties. In this study, it was found that all the specimens has shown their lowest in $\eta$. It is also seen that the modulus elasticity for compressive decreases as the volume fraction of the same type of microballoons increases as shown in Fig. 8 (b).

The specific compressive strength observed in Fig. 8 (c), show that the trend also reduces when more glass microballoons are added. The highest specific compressive strength still belongs to SCFT-01 at $86 \mathrm{MPa} / \mathrm{mgm}^{-3}$. This also reflects the 
lower $\eta$ and $\omega$ which decreases when specific compressive decreases. Therefore, even though SCFT-01 had a lower $\eta$, it still has a maximum $\omega$ for compressive showing that it is higher than neat resin. Hence, for compressive loading conditions, syntactic foams particularly SCFT-01 can lead to a significant advantage over the neat resin in terms of weight saving. A summary of the compressive results also can be seen in Table 1 .

The results also indicate that the compressive strength can be tailored over a wide range by selecting microballoons and by using them in different weight percentages. The maximum compressive strengths of the syntactic foams indicate a decrease when glass microballoons are added. The compressive stress for SCFT-01 is higher at $89 \mathrm{MPa}$ but still lower than pure vinyl ester with $112 \mathrm{MPa}$. While the modulus elasticity shows a decrease as well as specific compressive and specific modulus. Even though the specific compressive strength for SCFT-01 look same level with pure vinyl ester but it is lower approximately $5 \%$ form neat resin while for specific modulus compressive strength shows that it is higher $5 \%$ than pure resin. This indicates that by adding a small amount of glass microballoons particularly to SCFT-01 with $2 \mathrm{wt} . \%$ have a significant effect on the low density foams for weight saving application.

The representative SEM microstructure for the fractured surface tensile specimens SCFT-04 and SCFT-05 can be seen in Fig. 9 (a) and (b), respectively. It can be observed that microballoon fragments are not present in the syntactic foam because it was found that there are several glass microballoons damages between matrix resin internal syntactic foam. The tensile fracture mechanism seems to be mainly related to particle-matrix debonding and the fractured matrix resin can be seen clearly for the specimens with higher resin content as shown in the higher magnification micrographs presented in Fig. 9 (c) and (d). The fractured surface of the resin exemplifies crack formation and propagation and severe shear bands are also shown in this figure. This is a similar result to that which occurred between epoxy matrix resin and glass microballoons studied by Swetha and Kumar [30]. As a result, the pattern of propagation is that of twist hackle lines. Therefore, with a decrease in the volume fraction of the matrix resin in the materi- al, the strength of the composite is observed to decrease. It also shows that it contributes to the low density behaviour if the matrix is reduced in the syntactic foam.

\subsection{Compressive Fracture Analysis}

Fractured surfaces of the compressive specimens were studied in detail and it was observed that shear cracks originated from the edges under the bottom side of the specimen. The formation of shear crack in the matrix is represented as a drop in the stress value of the stress-strain curve. When the specimen is in the plateau region of the stress-strain curve, more shear bands are formed and barrelling of the specimen takes place. At a later stage, along with shear, axial splitting occurs and spalling of the specimen into small pieces is observed. For the specimens SCFT-01 with $2 \mathrm{wt} \%$ to SCFT-03 with $6 \mathrm{wt} \%$ of glass microballoons, the modes are barrelling along with shear yielding alone until crack in the middle of $10 \mathrm{wt} \%$ specimen is observed (schematic shown in Fig. 10) which is in agreement with earlier results [37]. Fig. 10 (a) represents the schematic of the sample when loaded, i.e., before the start of the stress-strain curve. The drop in the stress value in Fig. 11(c) and 11 (d) is due to initial crack formation which is shown in Fig. 10 (b).

Therefore, for densification strain, the glass microballoons enhance the densification strain of the foams. It is interesting that there is a slight reduction in energy while the variety of the foams increased with different contents of glass microballoon. It is observed that the foam specimens SCFT-01 containing 2wt.\% microballoons deform with a longer time to fracture as well as having higher stress behaviour at $89 \mathrm{MPa}$ compared to the other specimens. The limited contents of glass microballoons also enhanced the strength of the syntactic foams which can be seen in the SEM micrograph in Fig. 12 (a). It also shows the gaps between the glass microballoons closest to each other especially for specimen SCFT-03 containing $6 \mathrm{wt} . \%$ and SCFT-04 with $8 \mathrm{wt} . \%$. The failure features of these specimens are similar to those presented earlier [29], which include the initiation of shear cracks in the specimen and the formation of fragments from the side walls. Inclusion of a higher weight percentage of stiff glass microballoons in the relatively ductile matrix re- 
sults in increased brittleness of the composite.

It can also be observed that these specimens are comparable their compressive strength values and deformed with regular distribution of glass microballoons content. While SCFT-5 those containing 10 wt.\% glass microballoons occurred many fractured and debris of glass microballoons shown in Fig. 12 (b). The failure features of these specimens are similar to those presented previously [6], which includes the initiation of shear cracks in the specimen and the formation of fragments from the sidewalls. Inclusion of a higher weight percentage of stiff glass microballoons in a relatively ductile matrix results in increased brittleness of the composite. In the plateau region, the samples become barrel shaped and the schematic at the end of the stress-strain curve is represented in Fig. 10 (b) and Fig. 10 (c). Similar trends are also observed by Gupta et. al. $[6,33]$ and in epoxy based polymeric foams with different porosity levels subjected to quasi-static compression [38].

The specimen for 2 wt. \% shows the glass microballoons distributed randomly in the matrix resin. The amount of glass microballoons is also lesser when the density of syntactic foams decrease, while for the specimen with $4 \mathrm{wt} . \%$, the distribution of glass microballoons increases when the density is increased almost double when compared with 2 wt. $\%$, as shown in Table 1 . The specimens with 8 wt. $\%$ and 10 wt. $\%$ show that the glass microballoons are uniformly distributed around the matrix with increasing density. SEM photos (in Fig. 12) revealed these phenomenon.

The fracture surfaces of some of the glass microballoons and the vinyl ester matrix syntactic foams are shown in Fig. 12. In Fig. 12(a) and (b), it can be observed that extensive microballoon damage takes place during the compressive fracture of the material. Matrix material such as vinyl ester in Fig. 12(b) reveals that the matrix has also deformed severely. Plastic deformation marks can be observed on the entire matrix surface, including where the microballoons were embedded before fracture. Microballoons fracture in the form of small fragments, which are present all over the fracture surface. The fracture of microballoons and generation of debris has also been observed in epoxy matrix syntactic foams [39]. The plastic deformation marks observed in the matrix are considerably more se- vere in vinyl ester matrix foams because of the higher failure strain of these composites.

\section{Discussion}

\subsection{Relation of Young's Modulus with Par- ticle Parameters $(\omega, \eta)$}

In order to explain, for better understanding, the relationship between all the parameters for syntactic foam and modulus elasticity, the theoretical model for the tensile testing result is good to analyse [17]. Gupta et al. (2010) was found that several reports worked on numerous models to predict the modulus for composite materials but only a few report work for syntactic foam which is effect by attended of porosity enclosed inside of the glass microballoon contributed higher weight percentage or volume fraction [13, 34-37]. The dilute dispersion of glass microballoons in the matrix resin can be estimated by using the differentiation scheme. Numerical differentiation was proposed to estimate the elastic properties of the syntactic foam for higher composition [38]. In other words, experimentally the dilute dispersion of glass microballoon was extended to the composite for higher volume fraction.

This dilute dispersion can be achieved by adding a small quantity or incremental quantity of glass microballoons until it reaches the desired quantity. Before achieving this quantity, the composite material must put their condition as homogenous effective medium or also called as infinitely dilute dispersion particle [17]. This mechanism can be explained and determined by Gupta et al. (2010) in the Equation (4) and (5),

$$
\begin{aligned}
& \frac{d E}{E}=f_{E}\left(E_{b}, \phi_{b}, E_{m}, \phi_{m}, \eta\right) d w \\
& \frac{d \emptyset}{\emptyset}=f_{\emptyset}\left(E_{b}, \phi_{b}, E_{m}, \phi_{m}, \eta\right) d w
\end{aligned}
$$

Where $f_{E}$ is Young's Modulus function and $f_{\varphi}$ is Poison ratio's function consist of $E_{b}$ modulus elasticity of glass microballoon, $\varphi_{b}$ - Poisson's ratio of glass microballoon, $\mathrm{E}_{\mathrm{m}}$ - modulus elasticity of matrix resin, $\varphi_{m}$ - Poisson's ratio of matrix resin, $\eta$ - radius ratio of glass microballoon and 
$\mathrm{dw}$ - different composition. The above equation also assumed that there is space available when glass microballoons are added to the composite for the entire homogenous effective medium in regards will replaced the matrix resin cavity. As a result, the composition of composite materials will increase and volume will decrease due to the pre-existing particles. In order to enhance the understanding of Equation (4), Gupta et al. (2010) corrected and replaced the with dw/(1-w/ $\mathrm{w}_{\mathrm{m}}$ ) where $\mathrm{w}_{\mathrm{m}}$ is maximum packing volume fraction of particles and can be assumed as random packing factor 0.637 . When both equations were integrated with the initial values for all parameters, the Young's modulus for the composite can be determined. The relative modulus elasticity of vinyl ester syntactic foam respected to the modulus vinyl ester matrix resin have been implemented by Gupta et al. (2010) and it can be used also in this study.

In order to explain the relationship for these equations, it can be plotted the graph between $E / E_{m}$ on the $y$-axis versus (1- $\eta$ ) while on the $x-a x-$ is is wall thickness glass microballoon shows at Fig.13. In this study, the graph shows that the trend is non-linear for different weight percentages of glass microballoons and wall thickness for both batches $A$ and $B$. It can be observed that the relationship between both parameters is non-linear with relative modulus (E/Em) which relative maximum at 1.15 for batch $A$ and 1.01 for batch $B$ occurred for the weight percentage (8 - $10 \mathrm{wt} \%)$, respectively. Based on this result, it is followed the theoretical values of $\eta<0.955$ which is result in composites with relative modulus $(\mathrm{E} / \mathrm{Em})>1$ which proposed by Gupta et. al [17]. As the value of $(1-\eta)$ increases, the dependence of relative Young's modulus on the wall thickness decreases, that is, the slope of the curves also decreases. Hence, these results also support the value of $\omega$ and $\eta$ in Table 1 showing decreases as well.

It can be observed that the results match well for syntactic foams containing 2-6 wt.\% microballoons however, the predicted values are higher than the experimental results for foams containing 8 and 10 wt. $\%$ glass microballoons. It can be observed in Table 3 that, for syntactic foams containing 8 and $10 \mathrm{wt}$.\% glass microballoons, the void volume fraction is higher, which is not included in the model as well. Hence, at this composition the experimental results are lower than the predicted values. In other words, glass hollow particles with density approximately higher than $190 \mathrm{~kg} / \mathrm{m} 3$ (see Table 1) lead to syntactic foams stiffer than the vinyl ester neat resin material for any value of $w$.

\subsection{Difference between tensile and com- pressive moduli}

A comparison shows that the tensile modulus values at Fig. 8(b) are $70-80 \%$ higher than the compressive modulus values for the same type of syntactic foam. Such difference was also observed in previously published data $[10,24,25$, 27]. Two possible reasons are identified for such difference. The first reason is the particle-matrix interfacial de-bonding. Existence of any de-bonding causes a difference in the extent of stress transfer between particle and matrix. It has been shown in some recent studies that the tensile modulus is sensitive to the presence of de-bonding [24]. In comparison, under compressive loading condition de-bonding does not play an important role because the matrix is compressed on the particle and separation occurs only in a small region in the direction transverse to the applied load. De-bonding seems to be present in these composites as observed in Fig. 9. Therefore, the presence of de-bonding would result in an increase in the tensile modulus, which is similar to the experimental observations made in the present study.

The second reason that plays an important role is the possibility of particle fracture under compressive loading conditions. It can be observed, in Fig. 8(b), that compressive modulus measurements can be taken in the region where load is approximately 40-90 MPa. The mean plateau region for all specimens shows linearity except for that of pure vinyl ester. The trend for the mean plateau also make a different gradient from one specimen to another. One of the reasons for this trend is that if more glass microballoons are added, the linearity of the mean plateau shows greater flatness, for example specimens SCFT-04 and SCFT-05. The density analysis was conducted using a pycnometer machine with the Quantachrome model as presented in Table 1. It can be seen that the density is higher for the lower glass microballoon content and low density belongs to the higher glass microballoon content. The low density particles of higher glass microbal- 
loon content have thinner wall thickness and are weaker in comparison to the lower glass microballoon content. As a result the porosity will take place and fill in the broken glass microballoon space and also contribute to lower density when higher compressive loading is achieved. While beside of this phenomena, it was similar occurs to the void content too whereas took place the empty space and contribute to the lower density behaviour. In addition, the data on working pressure for glass microballoon survival are presented in Section 2.1 and show that only a maximum at 500psi which is equivalent to $3 \mathrm{MPa}$ when calculated. From these values, it appears that the fraction of weaker microballoons can potentially fracture in the measurement range of $40-90 \mathrm{MPa}$ under compressive stress-strain.

\subsection{Comparison of effect on mechanical} properties with other vinyl ester syntactic foams

The explanation on the comparison among this syntactic foam and another foams can be explained using the Ashby plot proposed by Thomas et. al [40] obtained from Granta Design software. In this study, it was plotted using Origin 8.0 software to make an easy way for the comparison. A comparison shows that the compressive strength of specimens from VE110 exhibited better performance when compared with previous strength of vinyl ester syntactic foams particularly specimen SCFT-01 as shown in Fig. 14. This result also shows that the magnitude bulk density used in this study given by the manufacturing company as $110 \mathrm{~kg} / \mathrm{m}^{3}$ has a lower density than other foams, such as $220 \mathrm{~kg} / \mathrm{m}^{3}, 370 \mathrm{~kg} /$ $\mathrm{m}^{3}$ and $460 \mathrm{~kg} / \mathrm{m}^{3}$. Therefore, true factor shows that the densities play a main role in achieving the higher compressive properties but that they are still lower than pure vinyl ester. Similarly, the tensile properties for this study are comparable with previous results for different types of vinyl syntactic foams. Figure 15 shows that the tensile strength for this study is marked as VE110 with the higher tensile modulus elasticity $40 \%$ higher than other syntactic foams, but still below the pure vinyl ester results. These results also can be seen in Fig. 8 (a) which is led by SCFT-01 particularly with $2 \mathrm{wt} . \%$ of glass microballoons. A similar trend was also observed where tensile strength and modulus elasticity syntactic foams are decreased when the volume fraction glass microballoons increased particularly for density
$220 \mathrm{~kg} / \mathrm{m}^{3}$ [36]. The low density glass microballoons shows higher compressive strength with the larger diameters and thinner walls thickness, and the higher density glass microballoons are weaker in tensile strength. In this study, the glass microballoons with density $110 \mathrm{~kg} / \mathrm{m}^{3}$ shows a good result for both the compressive and tensile strength results. While the modulus elasticity is that higher than other syntactic foams.

\section{Conclusions}

This detailed experimental study of the synthesis and characterisation of glass microballoon/ vinyl ester syntactic foam provides a number of findings. It might be useful to discovered and reported for all an important finding in the prediction and interpretation of the properties of glass microballoon/vinyl ester syntactic foam in this study:

- It is revealed that the density of syntactic foam varied and decreased while the glass microballoon contents increased, which followed the rule of mixture

- $\quad$ The parameters such as wall thickness, $\omega$ and radius ratio, $n$ play important roles in contributing to low density foam behaviour

- Porosity and void content were calculated and it was found that cavity porosity is higher than matrix porosity but void contents are remain constant in all specimens. This might be due to care taken during sample preparation and that gentle conventional stirring is enough to ensure that fewer glass microballoon are broken

- Tensile and compressive characteristics of the vinyl ester matrix syntactic foam are investigated and it is revealed that tensile strength is $70-80 \%$ higher than compressive when glass content is reduced

- Both of compression and tensile shows comparison result may be attributed to the measurement procedure and the possibility of particle fractured under compressive loading even at low load levels

- The maximum strength for both testing is led by SCFT-01 (2 wt.\%) glass microballoons. Even though SCFT-01 had a tensile modulus lower when compared to all specimens, it is still 
has a higher compressive modulus

- $\quad$ Compressive moduli of this foam is found to be lower than that of neat resin, but specific compressive modulus is higher

- $\quad$ Both the specific tensile and specific compressive had a higher value for SCFT-01 when compared with the other specimens which is useful for light weight material

- It is observed, from the relative modulus elasticity results, that the microballoons of $n<$ 0:955 are used in this study then the resulting syntactic foams would show substantial benefit in mechanical properties

- $\quad$ Both the compressive and tensile strength for VE110 vinyl/ester glass microballoons is led when compared with other glass microballoon types syntactic foams.

Hence, these beneficial results show that vinyl ester matrix syntactic foams have promise for structural application for their weight saving properties and that they might be can applied to marine structures.

\section{Acknowledgement}

The authors would like to thank Majlis Amanah Rakyat (MARA), Malaysia and Universiti Kuala Lumpur Malaysian Institute of Marine Engineering Technology, Malaysia for providing a schoarship to the first author of this work.

\section{References}

[1] Bardella L, El-Hadek MA, Tippur HV. Discussion on the paper "Simulation of porosity by microballoon dispersion in epoxy and urethane: Mechanical measurements and models. Journal of Materials Science Letters. 2003;22:1643-6.

[2] Gupta N, Ricci W. Comparison of compressive properties of layered syntactic foams having gradient in microballoon volume fractionand wall thickness. Materials Science and Engineering A. 2006;427:331-42.

[3] Park S, Jin F, Lee C. Preparation and physical properties of hollow glass microspheres-reinforced epoxy matrix resins. Material Science Engineering A. 2005;402:335-40.

[4] Tien CL, Gupta N, Talalayev A. Thermoanalytical characterization of epoxy matrix-glass microbal-
Ioon syntactic foams. Journal Material Science. 2009;44:1520-7.

[5] Vasanth C, Dinesh P, Gupta N. Thermal expansion behaviour of hollow glass particle/vinyl ester composites. Journal Materials Science. 2012;47:5596-604.

[6] Gupta N, Woldesenbet E, Kishore, Sankaran S. Studies on Compressive Failure Features in Syntactic Foam Material. Journals of Sandwich Structures and Materials. 2001;4:249-72.

[7] SALLEH Z, ISLAM, M.M. and KU, H. Study on Compressive Properties of Syntactic Foams for Marine Applications<Paper Syntactic Foam-zulzamri.pdf>. Journal of Multifunctional Composite. 2014:21-7.

[8] Gupta N, Priya S, Islam R, Ricci W. Characterization of mechanical and electrical properties of epoxy-glass microballoon syntactic composites. Ferroelectrics. 2006;345:1-12.

[9] Wouterson EM, Boey FYC, Hu X, Wong SC. Effect of fiber reinforcement on the tensile, fracture and thermal properties of syntactic foam. Polymer. 2007;48:3183-91.

[10] Kishore, Shankar R, Sankaran. Gradient syntactic foams: Tensile strength, modulus and fractographic features. Materials Science and Engineering A. 2005;412:153-8.

[11] Karthikeyan C, Sankaran S, Kumar M, Kishore. Processing and compressive strengths of syntactic foams with and without fibrous reinforcements. Journal of Applied Polymer Science 2001;81:40511.

[12] Wouterson E, Boey F, Hu X, Wong S. Fracture and impact toughness of syntactic foam. Journal of Cell Plastics. 2004;40:145-54.

[13] Bardella L, Genna F. Elastic design of syntactic foamed sandwiches obtained by filling of three-dimensional sandwich-fabric panel. International Journal of Solids and Structures. 2001;38:307-33.

[14] Bardella L, Genna F. Elastic design of syntactic foamed sandwiches obtained by filling of three-dimensional sandwich-fabric panels. International Journal of Solids and Structures. 2001a;38:30733.

[15] Sagi-Mana D, Narkis M, Siegmann A, Joseph R, Dodiuk $\mathrm{H}$. The effect of marine environment on a vinyl ester resin and its highly filled particulate quartz composites. Journal Applied Polymer Science 1998;69:2229-34.

[16] Rajapakse Y, Hui D. Marine composites and sandwich structures. Composites B Engineering. 2008;39:1-4.

[17] Gupta N, Ye R, Porfiri M. Comparison of tensile 
and compressive characteristics of vinyl ester/ glass microballoon syntactic foams. Composites Part B: Engineering. 2010;41:236-45.

[18] Bunn P, Mottram JT. Manufacture and Compression Properties of Syntactic Foams. Composites Part A: Applied Science and Manufacturing. 1993;24:565-71.

[19] Balch D, Dunand D. Load partitioning in aluminum syntactic foams containing ceramic microspheres. . Acta Material 2006;54:1501-11.

[20] Imre NO, Kornél M. Microballoon of Metal-matrix composite reinforced by ceramic microballoon. Physics of Fluids. 2004;16:11180-1126.

[21] Palmer RA, Gao K, Doan TM, Green L, Cavallaro G. Pressure infiltrated syntactic foams-Process development and mechanical properties. Materials Science and Engineering: A. 2007;464:85-92.

[22] Hussain M, Nakahira A, Nishijima S, Niihara K. Fracture behaviour and fracture toughness of particulate filled epoxy composites. Material Letter 1996;27:21-5.

[23] Broutman L, Sahu S. The effect of interfacial bonding on the toughness of glass filled polymers. Material Science Engineering 1971;8:98-107.

[24] Tagliavia G, Porfiri M, Gupta N. Vinyl ester-glass hollow particle composites: dynamic mechanical properties at high inclusion volume fraction. Journal Composite Material. 2009;43:561-82.

[25] Gupta N, Nagorny R. Tensile properties of glass microballoon-epoxy resin syntactic foams. Journal of Applied Polymer Science. 2006;102:1254-61.

[26] Islam MM, Kim HS. Novel syntactic foams made of ceramic hollow micro-spheres and starch: theory, structure and properties. Journal of Materials Science. 2007;42:6123-32.

[27] Gupta N, Woldesenbet E, Mensah P. Compression Properties of Syntactic Foams: Effect of Cenosphere Radius Ratio and Specimen Aspect Ratio. Composites: Part A. 2004;35:103-11.

[28] Division PILEGM. Spherical Typical Product Characteristics Valley Forge, PA 19482 USA: Potters Industries LLC; 2011.

[29] John B, Nair CPR, Devi KA, Ninan KN. Effect of low-density filler on mechanical properties of syntactic foams of cyanate ester. Journal of Materials Science. 2007;42:5398-405.

[30] Swetha C, Kumar R. Quasi-static uni-axial compression behaviour of hollow glass microspheres/ epoxy based syntactic foams. Materials \& Design. 2011;32:4152-63.

[31] Phang Z, Ding J. Poly(lactide-co-glycolide) porous scaffolds for tissue engineering and regener- ative medicine. Interface Focus. 2012;2:366-77.

[32] Kim HS, Plubrai P. Manufacturing and failure mechanisms of syntactic foam under compression $\square$. Composites Part A: Applied Science and Manufacturing. 2004;35:1009-15.

[33] G Subhasha, Q Liu, Gao XL. Quas static uni axial compression behaviour of hollow glass microspheres epoxy based syntactic foams. International Journal Impact Engineering 2006;32:1113-26.

[34] M. Koopman, K. Chawla, N. Carlisle N, Gladysz. G. Microstructural failure modes in three-phase glass syntactic foams. Journal of Material Science 2006;41:4009-14.

[35] Gladysz G, Perry B, McEachen G, Lula J. Three-phase syntactic foams: structure-property relationships. Journal of Material Science 2006;41:4085-92.

[36] Huang JS, Gibson LJ. Elastic moduli of a composite of hollow spheres in a matrix. . Journal Mechanic Physics Solids 1993;41:55-75.

[37] Li P, Petrinic N, Siviour CR, Froud R, Reed. Strain rate dependent compressive properties of glass-microbaloon epoxy syntactic foams. Journal of Material Science Engineering A 2009;515:1925.

[38] C. Periasamy, Jhaver R, Tippur HV. Quasi-static and dynamic compression response of a light weight interpenetrating phase composite foam. Material Science Engineering A. 2010;527:284556.

[39] Gupta N, Woldesenbet E, Kishore. Compressive fracture features of syntactic foams-microscopic examination. Journal Material Science. 2002;37:3199-31209.

[40] Thomas H, Ling L, Wong C, Koon Y, Alexander B. Tailored for simplicity: creating high porosity, high performance bio-based macroporous polymers from foam templates. The Royal Society of Chemistry. 2013;16:1931-40.

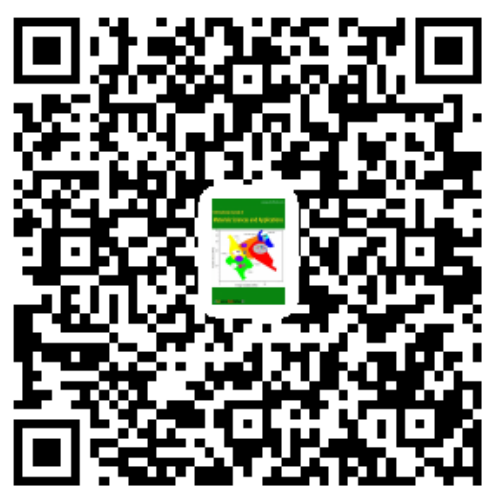




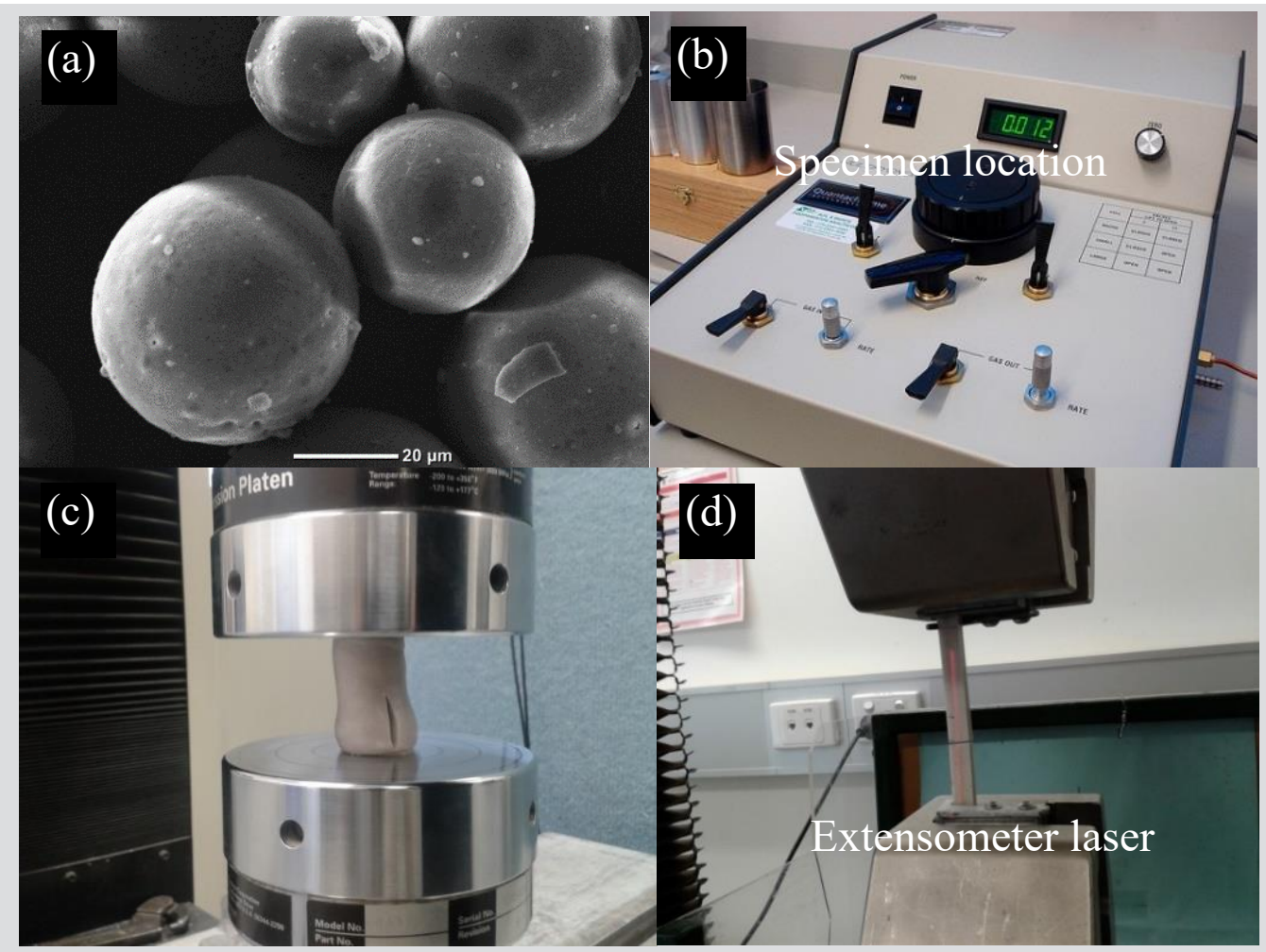

Fig. 1. (a) A SEM photo for particles VE1 10 glass microballoons and related equipment used in this study (b) Multipycnometer unit (c) MTS Insight compression machine (d) MTS Insight universal tensile machine.
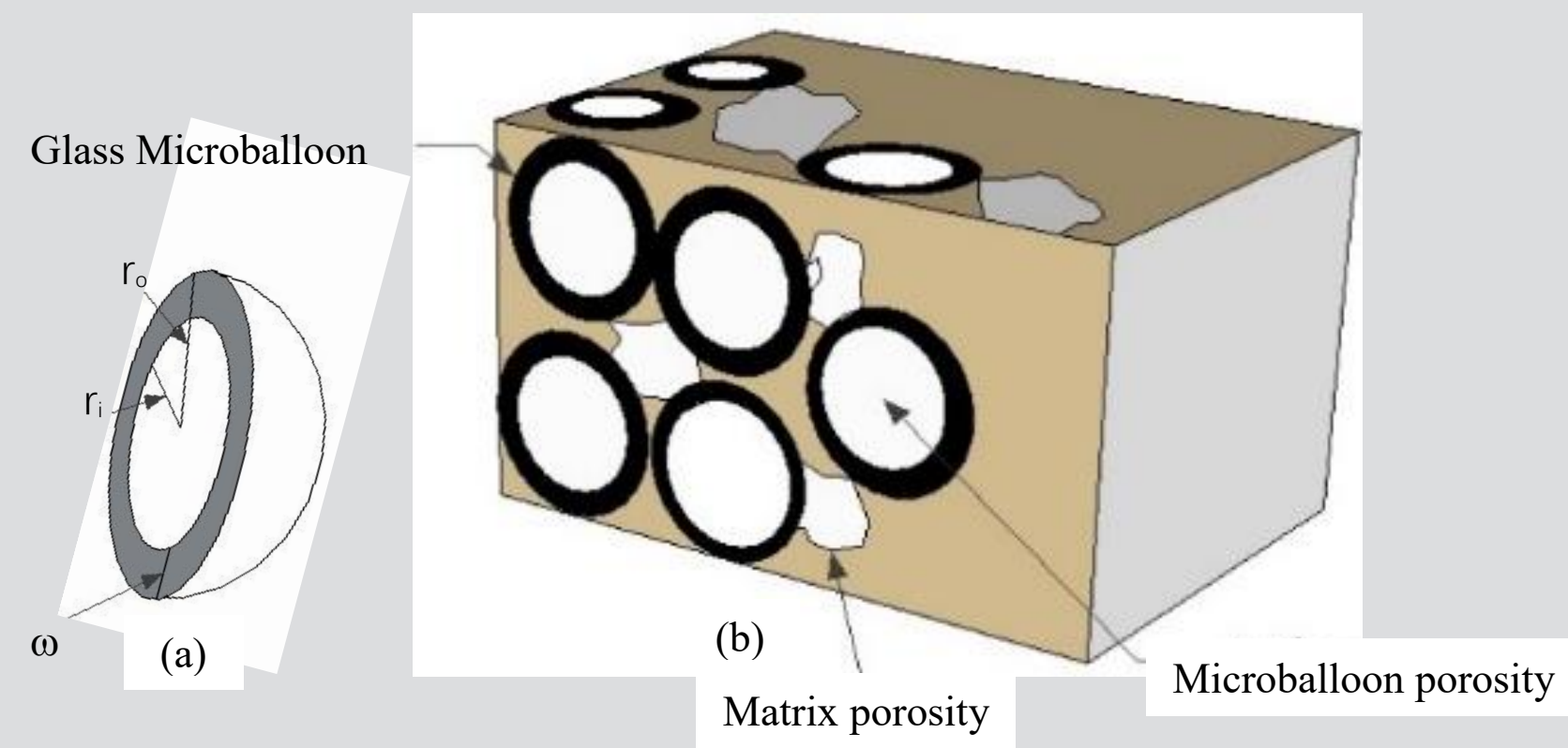

Fig.2. Schematic diagram for structure of (a) Glass microballoon (b) Syntactic foams showing microballoons with porosities 


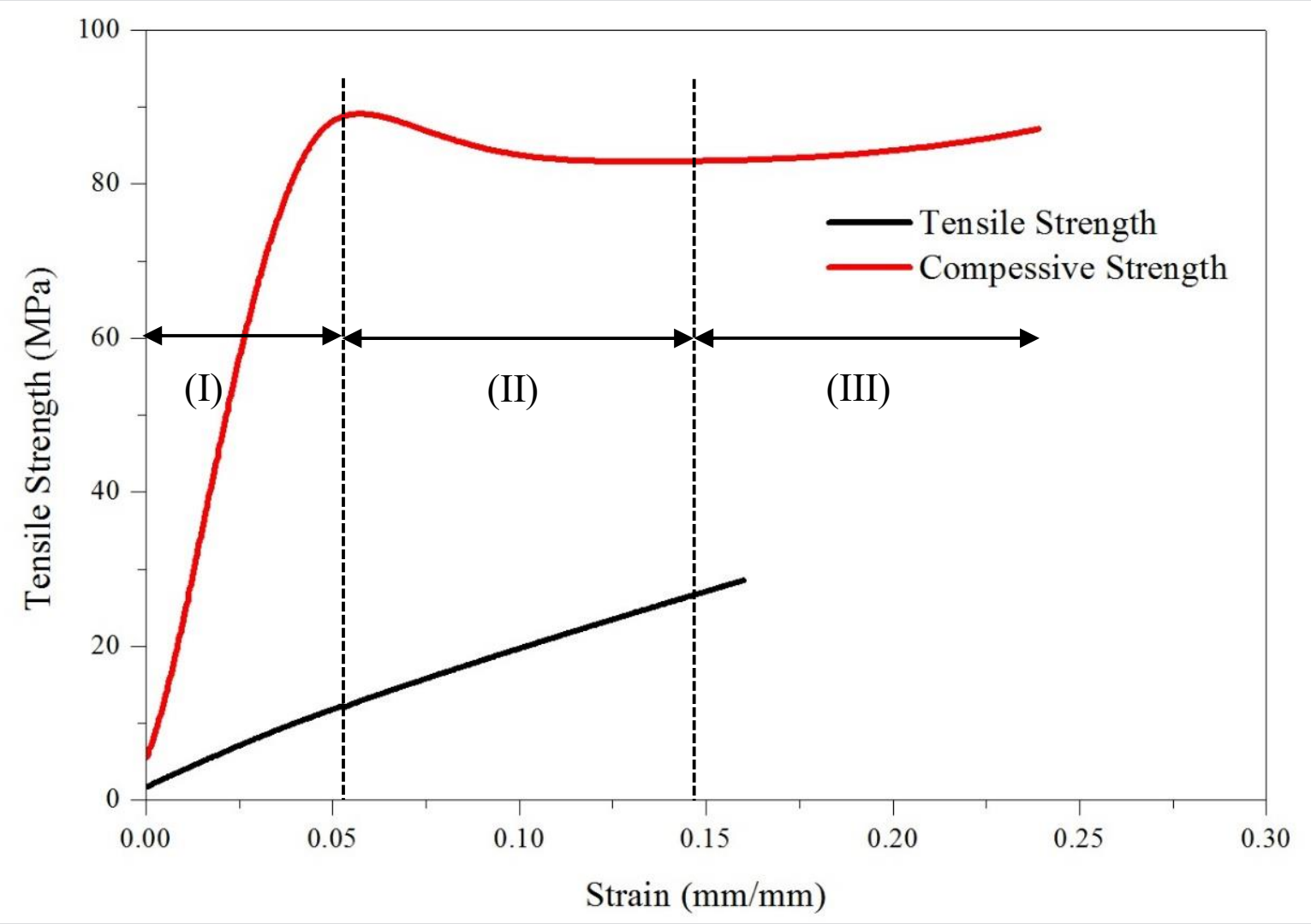

Fig. 3. Typical curve of a stress-strain for tensile and compression of syntactic foam.

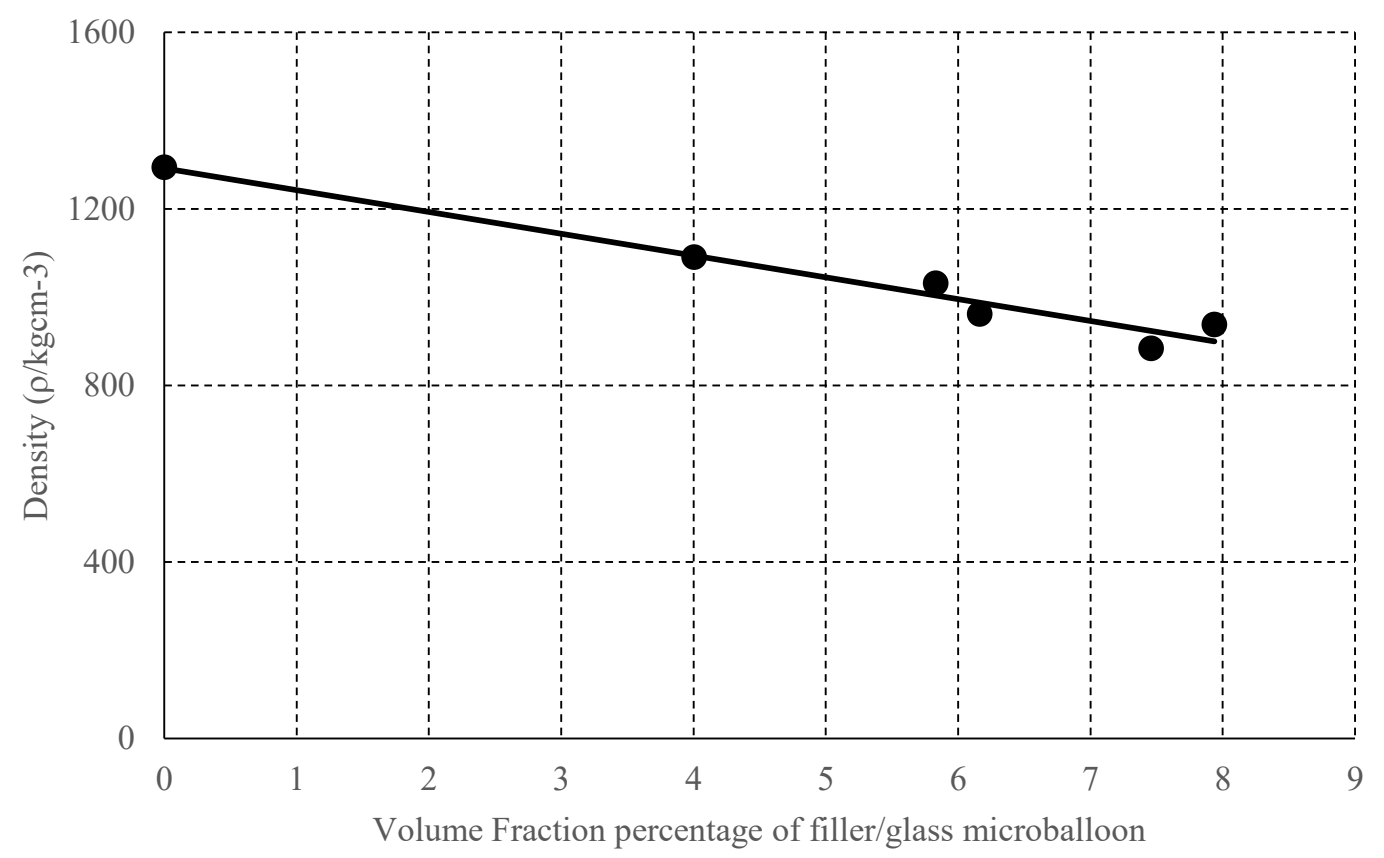

Fig. 4. Density of syntactic foam as a function of volume fraction of glass microballoon exhibiting a linear trend as per the rule of mixtures. 

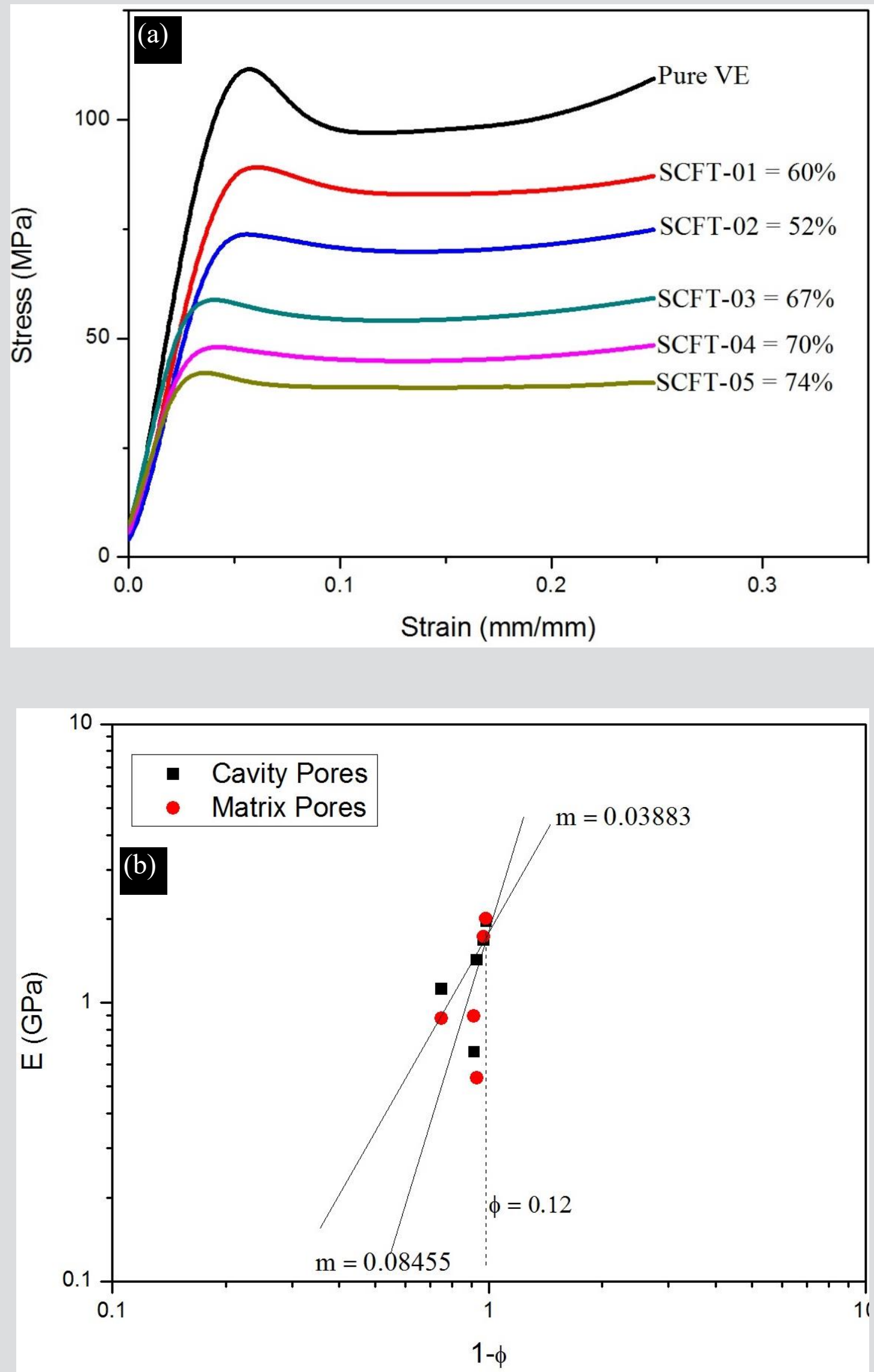

Fig. 5. Compressive strength related to porosities of syntactic foam a) Total porosity (b) Compressive modulus related with relative density. 

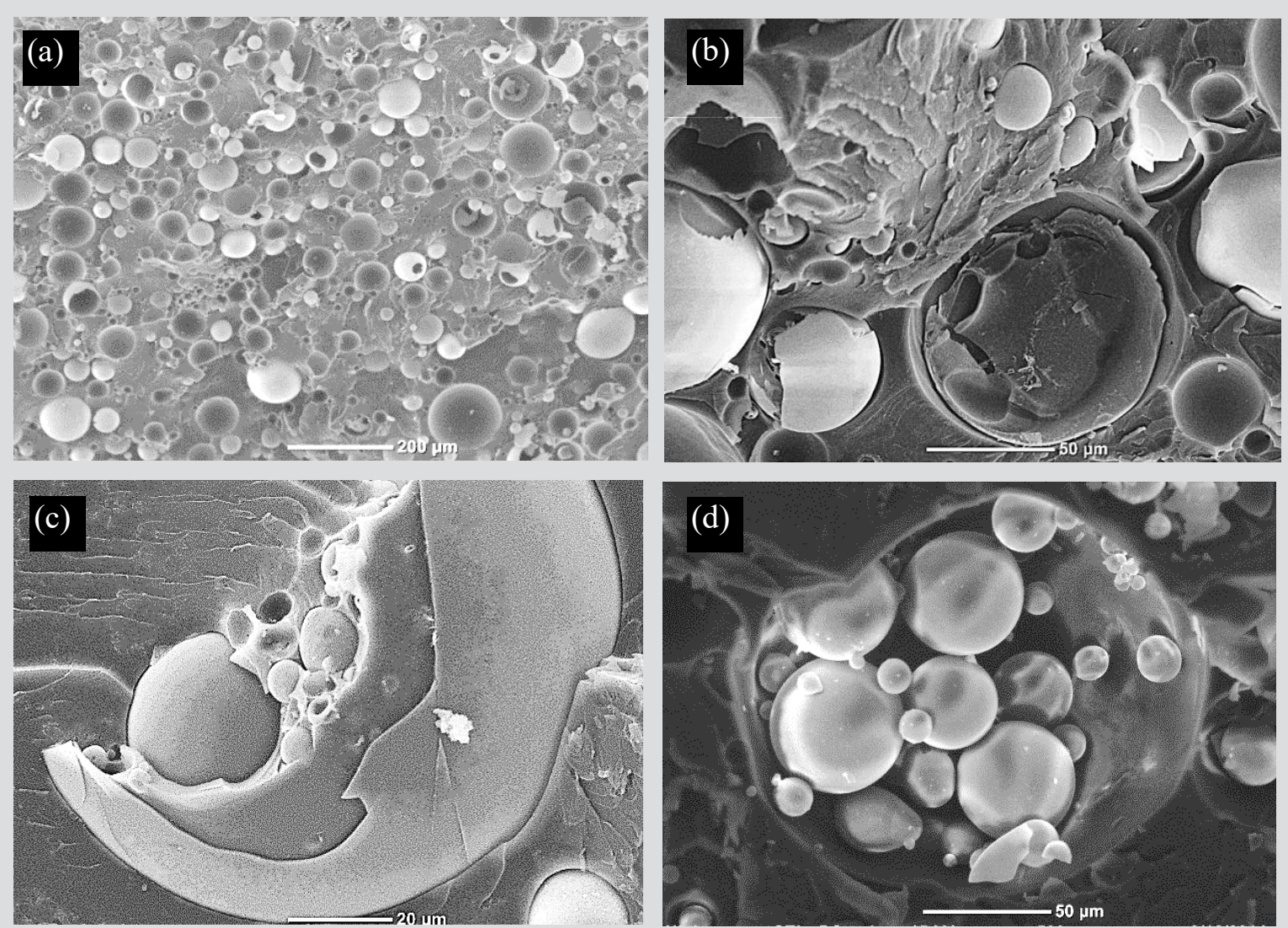

Fig. 6. A SEM showing (a) Two types of porosity cavities and matrix porosities for SCFT-04 (8 wt.\%) (b) Air entrapped for SCFT-02 (4 wt.\%) (c) Filled with resin for SCFT-01(2 wt.\%) (d) Filled with small glass microballoons for SCFT-02(4 wt.\%)
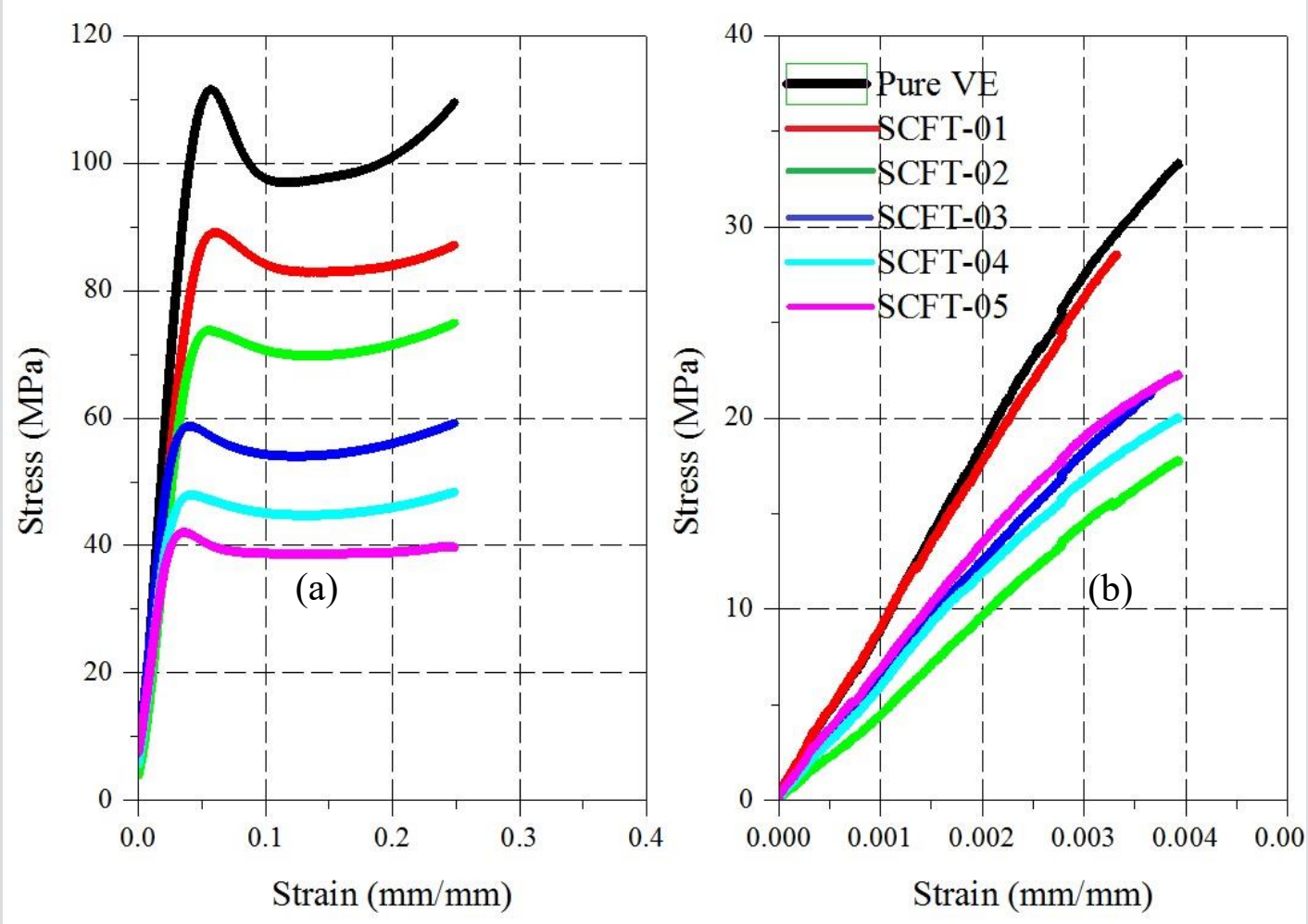

Fig. 7. Representative mechanical strength curve for vinyl ester matrix syntactic foam (a) Compression (b) Tensile. 


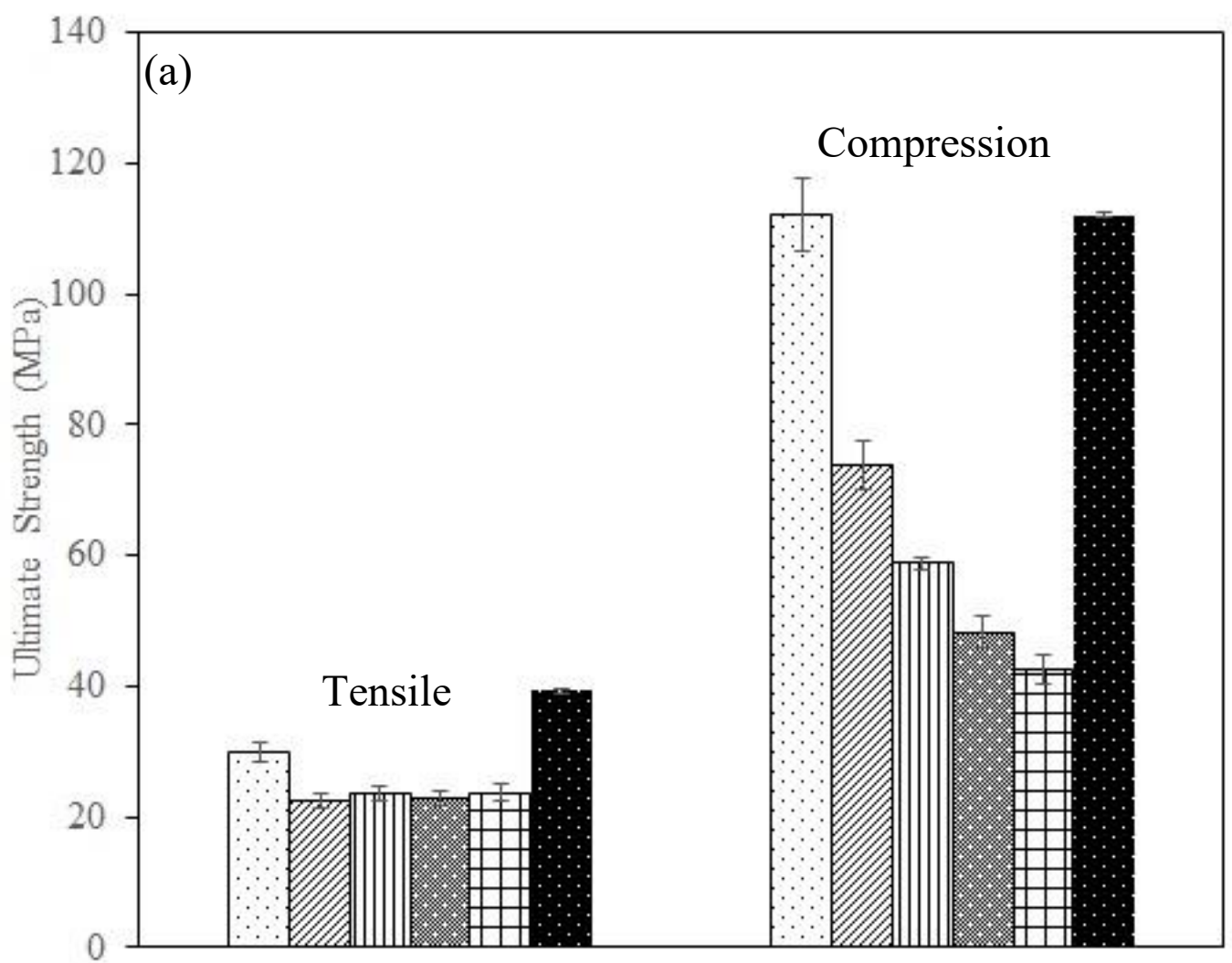

口SCFT -01

øSCFT -02

m SCFT -03

图 SCFT -04

口SCFT -05

- Pure VE

Glass microballoon specimens

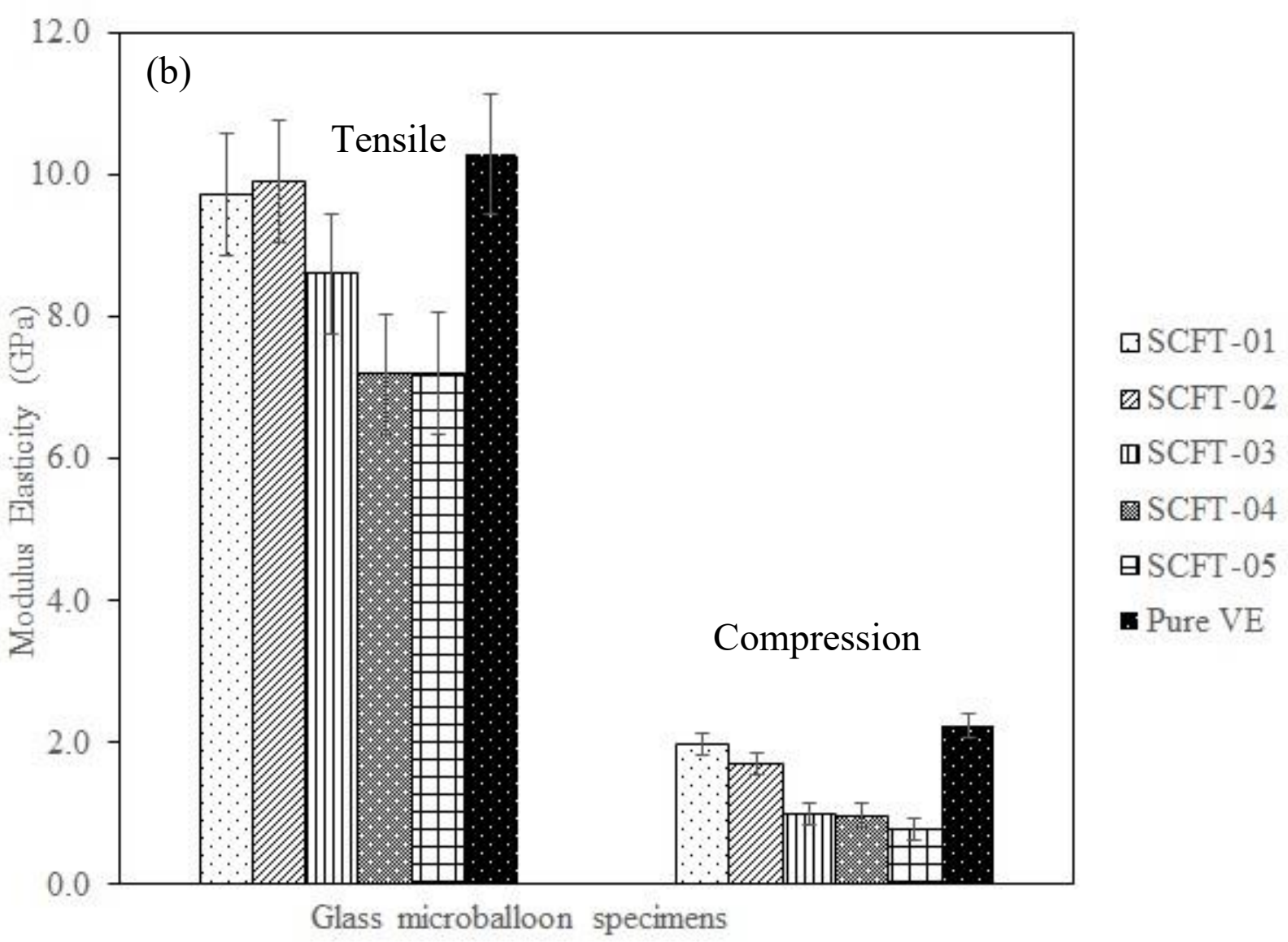




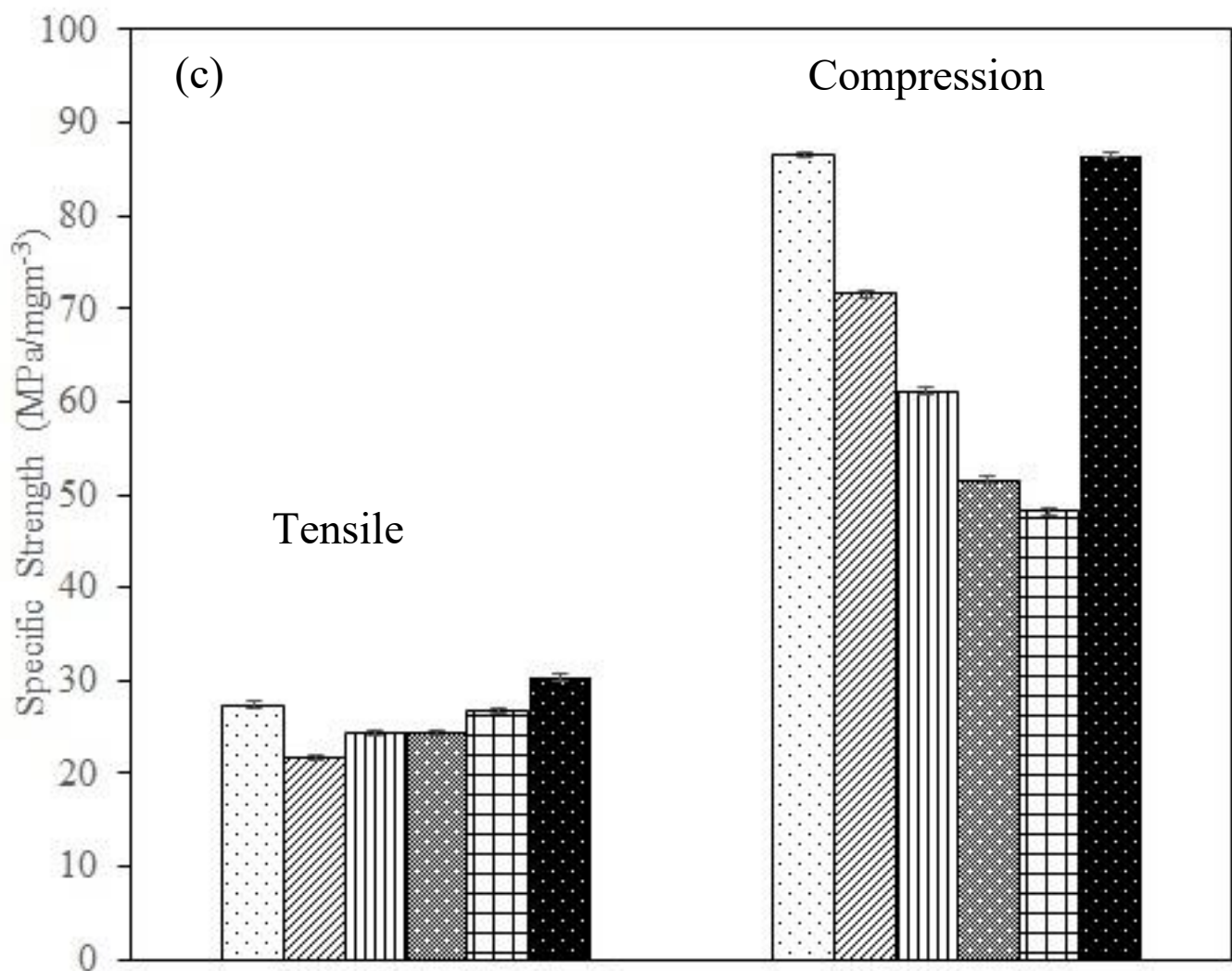

Glass microballoon specimens

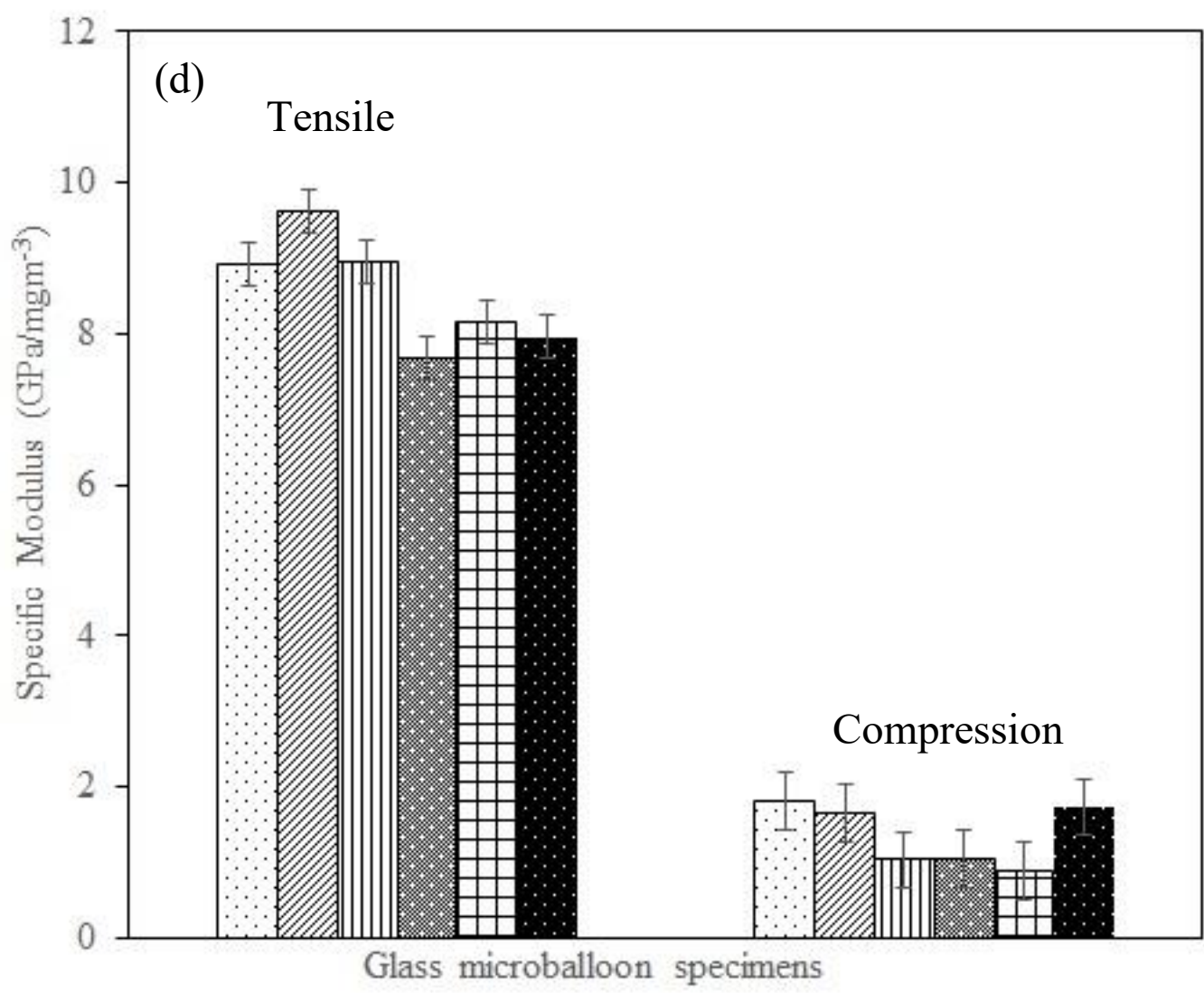

口SCFT - 01

ÐSCFT -02

m SCFT -03

圆 SCFT -04

口SCFT-05

. Pure VE

Fig. 8. Comparison of representative tensile and compression strength with different weight percentage of glass microballoon for (a) Tensile Max. (b) Modulus Elasticity (c) Specific Tensile (d) Specific Modulus. 

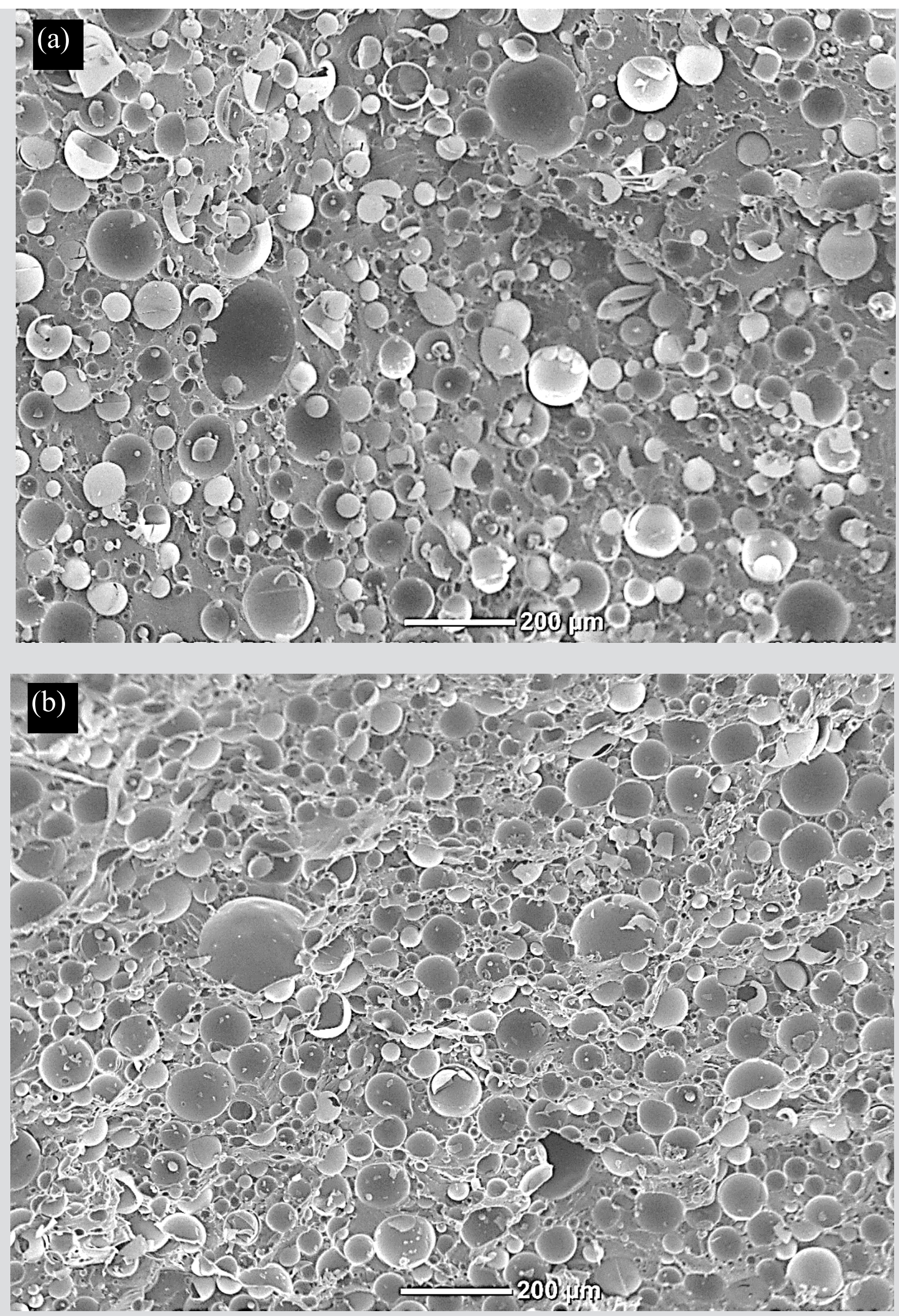

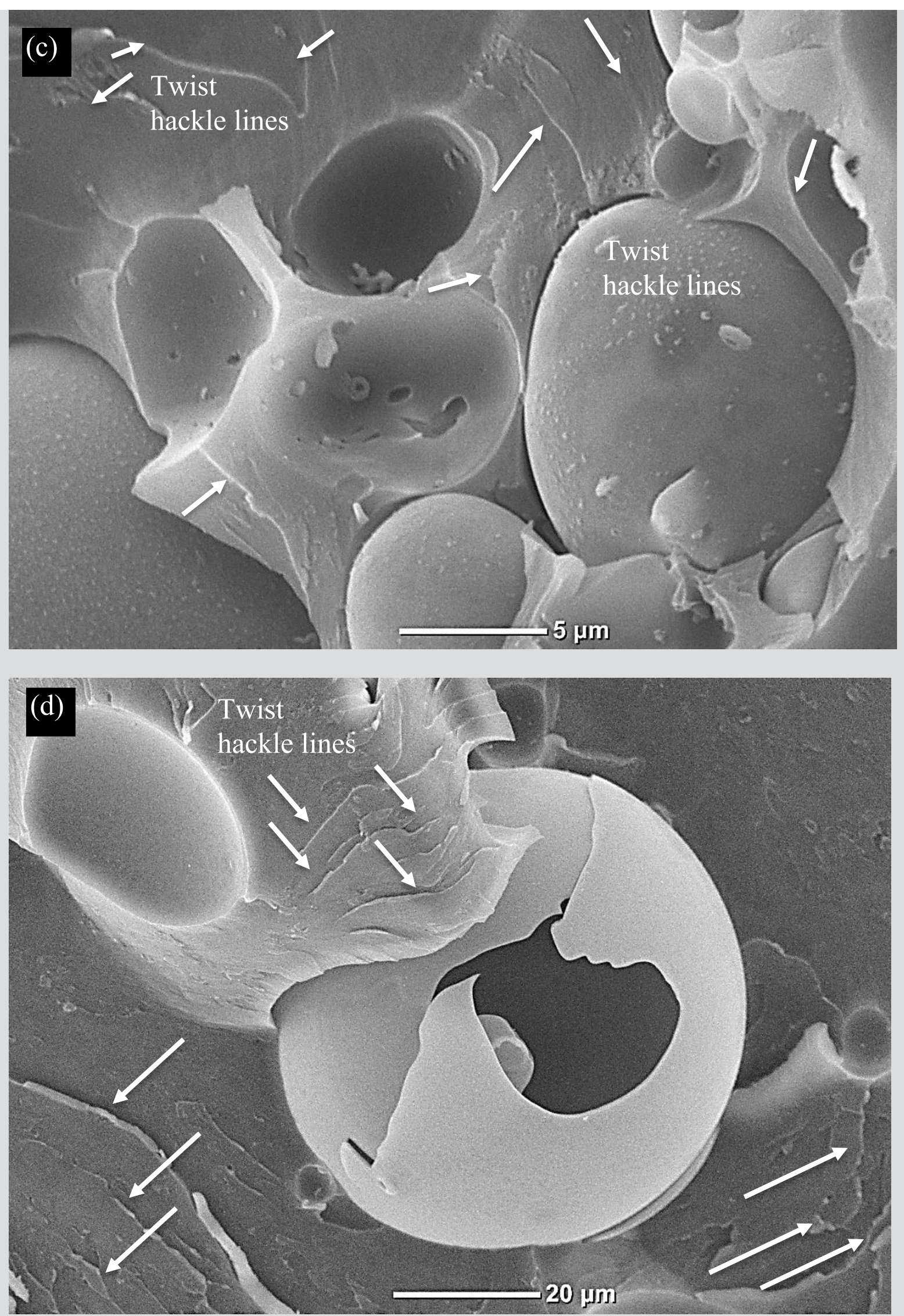

Fig. 9. Representative of tensile SEM microstructure for (a) SCFT-04 (b) SCFT-05 (c) SCFT-1 (d) SCFT-3 at higher magnification micrograph 

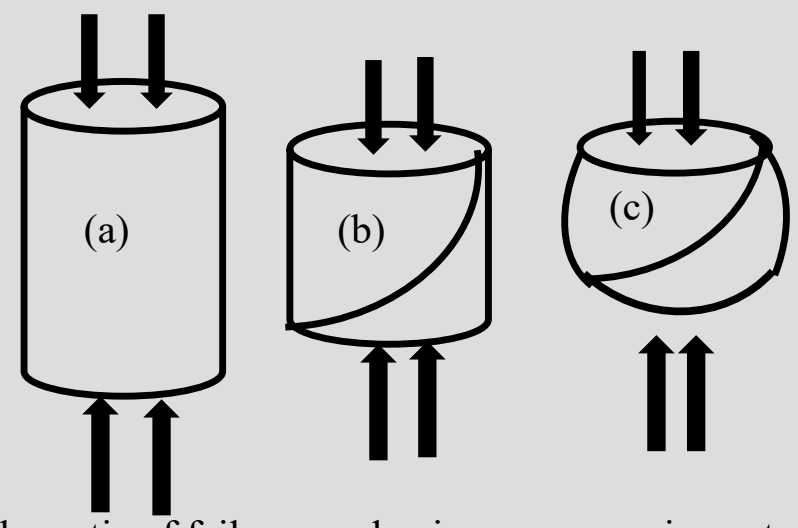

Fig.10. Schematic of failure mechanism sequence in syntactic foams (a) Initial stress

(b) Internal crack stress concentration (c) Barreling shape situation.
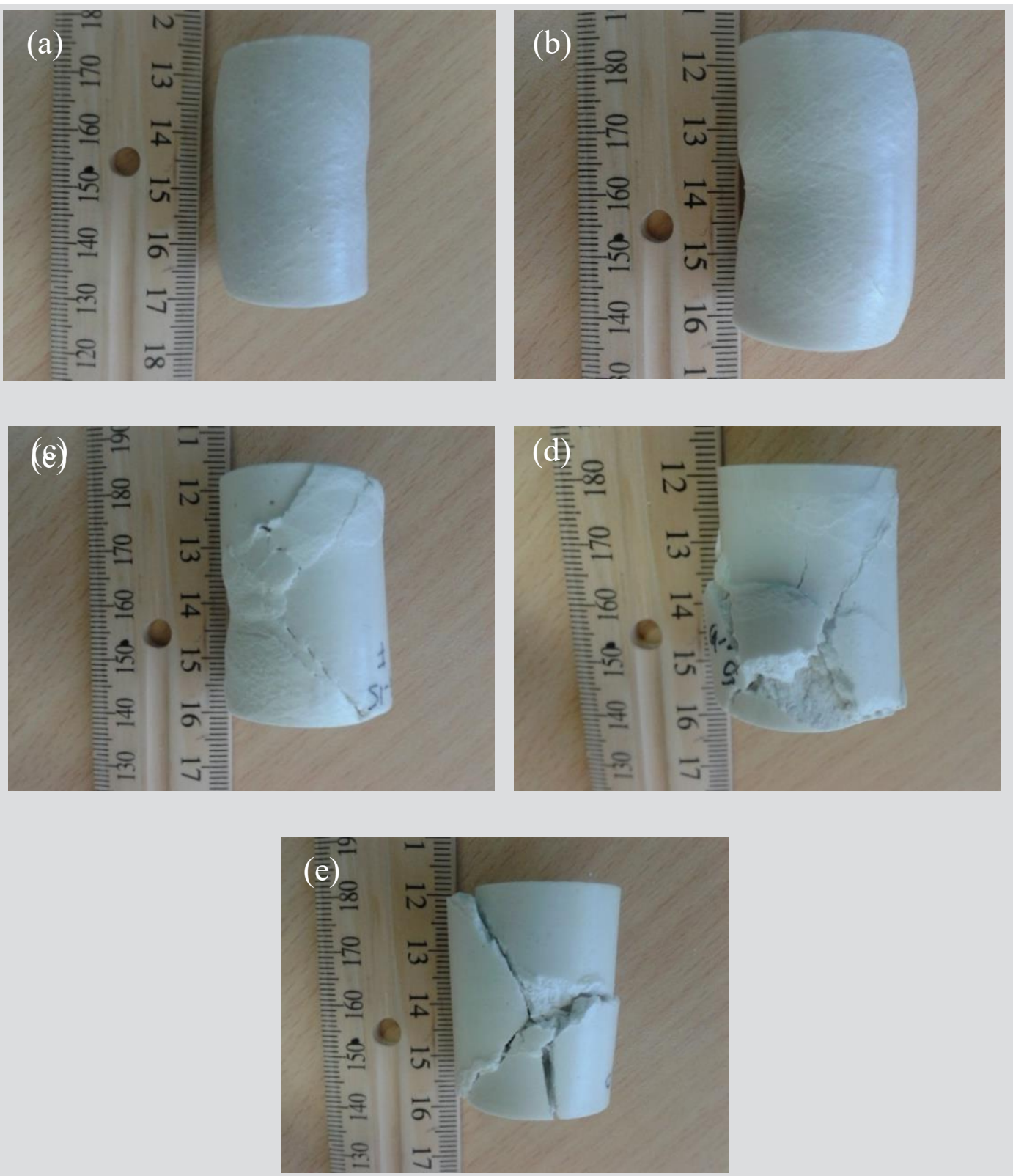

Fig. 11. Representative specimens of failure mechanism sequence in syntactic foam (a) SCFT-01 (b) SCFT-02 have barrel shape failure mode (c) SCFT-03 (d) SCFT-04 and SCFT-05 have spalling of syntactic foam 

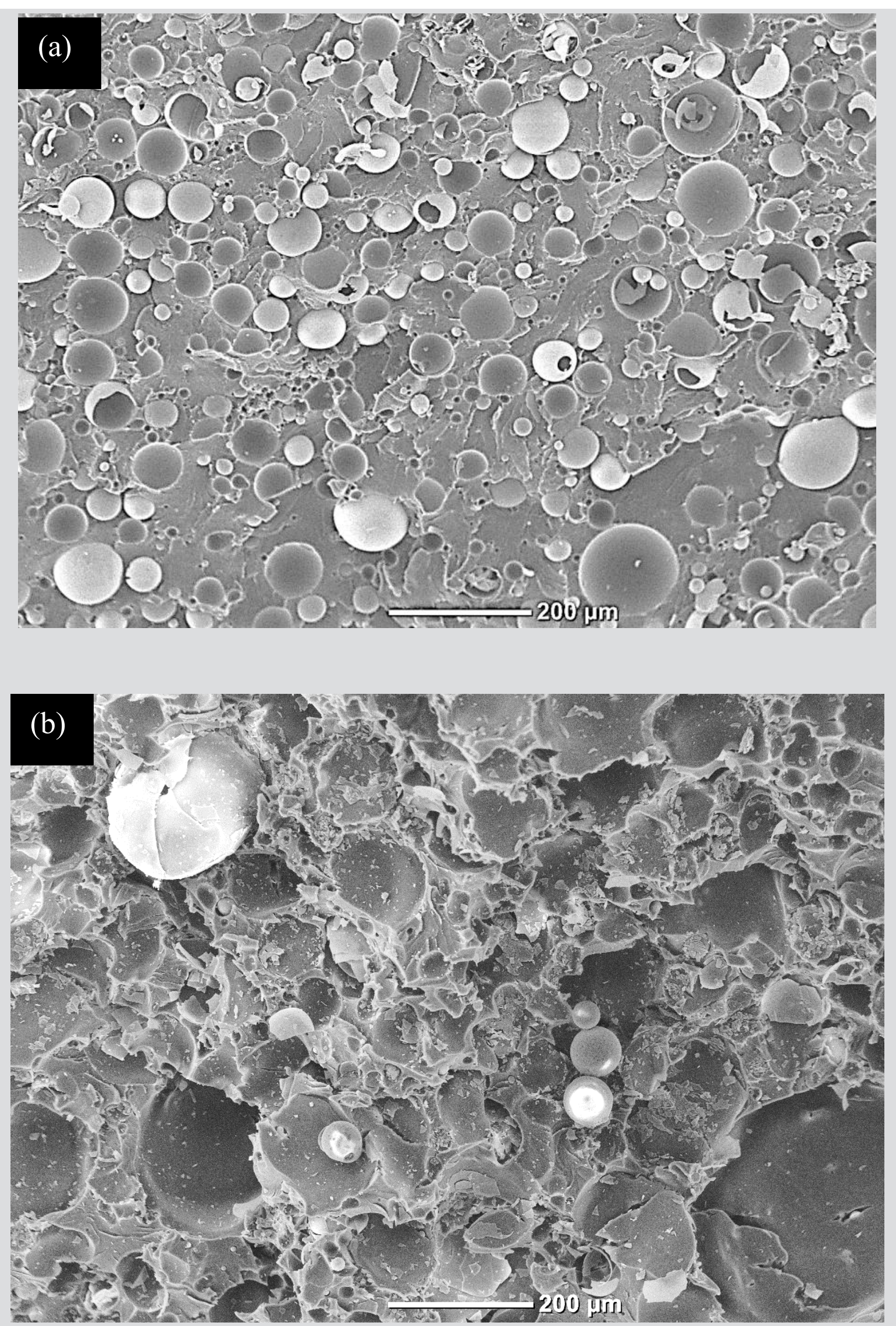

Fig. 12. Representative fracture surface of (a) SCFT-04 (glass microballoon 8wt.\%) (b) SCFT-05 (glass microballoon $10 \mathrm{wt} . \%$ ). 


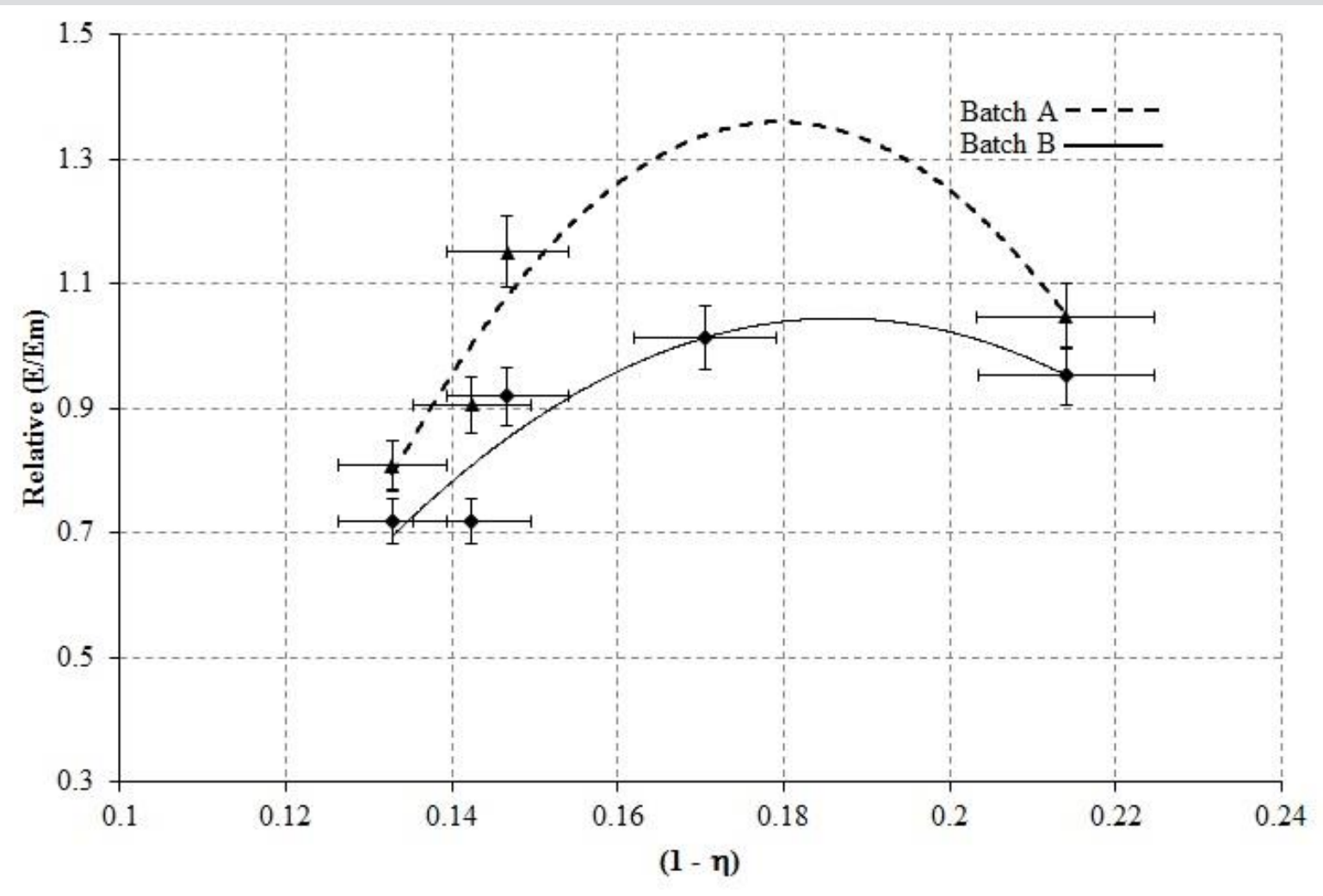

Fig. 13. Relationship between Young's modulus (E/Em) and particle

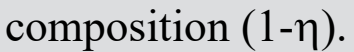
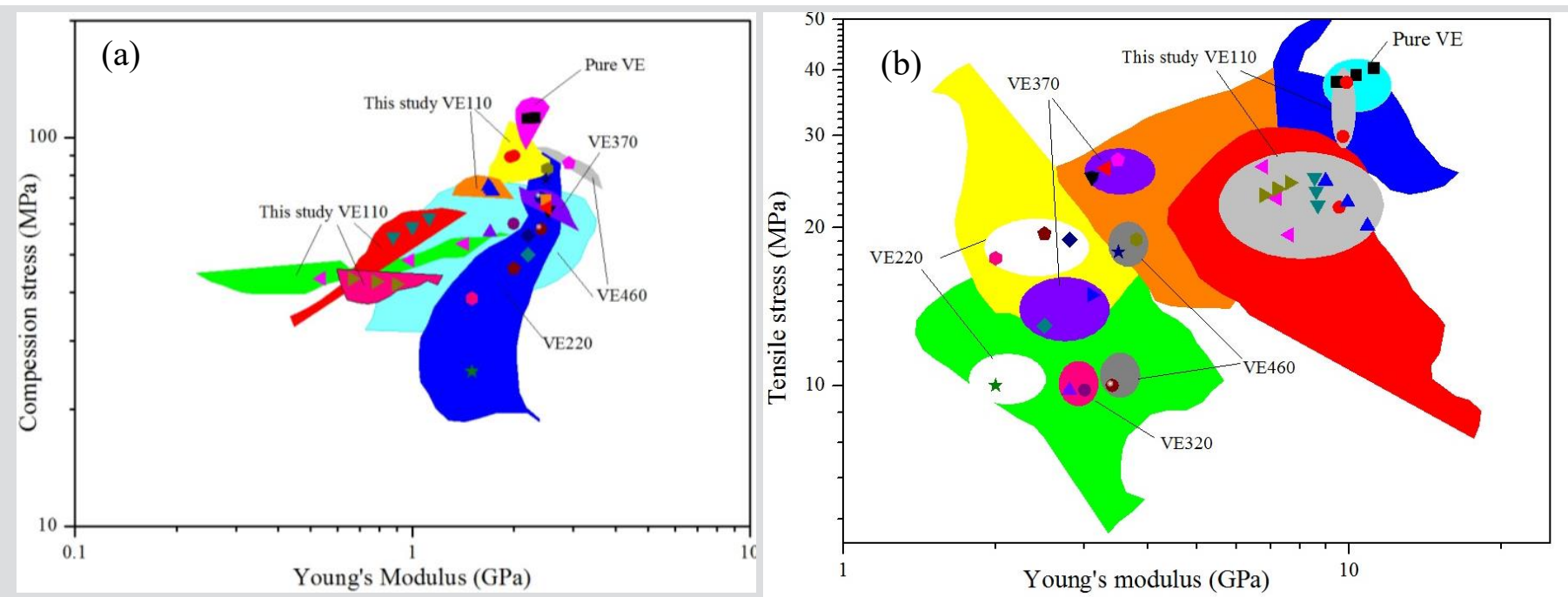

Fig. 14. A comparison for (a) Compressive strength (b) Tensile strength of vinyl ester syntactic foam in this study and various different types of syntactic foam [17] . 